\title{
Searching for light WIMPS via their interaction with electrons
}

\author{
J.D. Vergados \\ University of Ioannina, Ioannina, Gr 451 10, Greece.
}

(Dated: May 15, 2020)

\begin{abstract}
In the present work we examine the possibility of detecting light dark matter particles in the few $\mathrm{MeV}$ region via their interactions with electrons. We analyze theoretically some key issues involved in such a detection and perform calculations for the expected rates, for electron recoils as well as spin induced atomic excitations, in the context of reasonable theoretical models.
\end{abstract}

PACS numbers: 93.35.+d 98.35.Gi 21.60.Cs

\section{INTRODUCTION}

The combined earlier results MAXIMA-1 [1], 2], 3], BOOMERANG [4], [5] DASI [ [6] and COBE/DMR Cosmic Microwave Background (CMB) observations [7], 8] imply that the Universe is flat [9] and that most of the matter in the universe is Dark [8]. These results have been confirmed and improved by the WMAP [10] and Planck data [11]. Combining these data one finds:

$$
\Omega_{b}=0.04867 \pm 0.00062, \quad \Omega_{\mathrm{CDM}}=0.26880 \pm 0.01152, \quad \Omega_{\Lambda}=0.685_{-0.016}^{+0.018}
$$

On the smaller scales there exists firm indirect evidence from the observed rotational curves, see e.g. the review [12], for a halo of dark matter in galaxies and dwarf galaxies.

Anyway in spite of the above indirect evidence for the existence of dark matter at all scales, it is essential to directly detect such matter in order to unravel the nature of its constituents.

It is clear that the direct detection of dark matter depends on the nature of the dark matter constituents and their interactions.

Historically the first dark matter particles considered were the WIMP's (Weakly interacting massive particles). Given the importance of dark matter, the dominant matter component in the universe, there is strong motivation to explore a broader set of dark matter candidates, beyond those of WIMPs, i.e. beyond candidates that interact with ordinary matter with essentially weak interactions. All such possibilities are currently examined, see e.g [13] and references there in, a white paper summarizing the workshop U.S. Cosmic Visions: New Ideas in Dark Matter, which calls out the importance of searching for dark matter along every feasible avenue.

Searching for WIMPs, however, still remains the most active field of research. WIMPs are expected to have a velocity distribution with an average velocity, close to the rotational velocity $v_{0}=220 \mathrm{~km} / \mathrm{s}$ of the Sun around the galaxy, i.e. they are completely non relativistic. In fact a Maxwell-Boltzmann distribution with a maximum cut off of about $2.84 v_{0}$ leads to a maximum energy transfer close to the average WIMP kinetic energy $\langle T\rangle \approx 0.4 \times 10^{-6} m c^{2}$. Thus for GeV WIMPS this average is in the keV regime, not high enough to excite the nucleus, with only 4 exceptions ${ }^{1}$, but sufficient to measure the nuclear recoil energy.

For light dark matter particles in the $\mathrm{MeV}$ region, which we will also call WIMPs, the average energy that can be transferred is in the eV region.

In the present work we will focus on light WIMPs. WIMPs with masses below the electron mass can only be detected by special materials involving essentially free electrons, like superconductors, by measuring the total deposited energy.

1 The exceptions are odd nuclei, with low lying excited states, which can be populated via a Gamow-Teller like excitation, and have been studied theoretically [14], 15] and references there in. These are: a) transition $5 / 2^{+} \rightarrow 7 / 2^{+}$to the first excited state at $57.7 \mathrm{keV}$ of ${ }^{127} \mathrm{I}$. b) transition $1 / 2^{+} \rightarrow 3 / 2^{+}$to the first excited state at $39.6 \mathrm{keV}$ of ${ }^{129} \mathrm{Xe}$. c) Transition $1 / 2^{+} \rightarrow 3 / 2^{+}$to the first excited state at $35.48 \mathrm{keV}$ of ${ }^{125} \mathrm{Te}$. d) Transition $9 / 2^{+} \rightarrow 7 / 2^{+}$to the first excited state at $9.4 \mathrm{keV}$ of ${ }^{83} \mathrm{Kr}$. Such transitions are due to the spin induced WIMP nucleus cross section. None has been observed. The same is true for the recoil experiments, which are due to the spin independent cross section 
Heavier WIMPS with a mass less than 50 times the electron mass can be detected by measuring the electron recoil, following the WIMP-electron interaction, in the case of some targets that posses weakly bound electrons. They can also be detected by inducing atomic excitations.

The event rate for such a process can be computed from the following ingredients [16]: i) The elementary WIMPelectron cross section. ii) The WIMP density in our vicinity obtained from the rotation curves. This yields a large number density due to the assumed smallness of the WIMP mass, expected to be about six orders of magnitude larger than that involved in the usual WIMPs considered in nuclear recoils. iii) The WIMP velocity distribution. In the present work we will consider a Maxwell-Boltzmann (MB) distribution in the galactic frame, with the WIMP velocity appropriately transformed in the local frame.

In all recoil experiments, like the nuclear measurements first proposed more than 30 years ago [17], in order to overcome the formidable background problems one can exploit the modulation effect, a periodic signal due to the motion of the earth around the Sun. Unfortunately this effect, also proposed a long time ago [18] and subsequently studied by many authors [16, 19 27], was found to be small in the case of nuclear recoils. We expect it to be larger in the case of electron recoils.

In spite of these problems many experiments undertook the task of detecting nuclear recoils in WIMP-nucleus scattering, see e.g. [28 37]. None has been detected but very stringent limits on the nucleon cross section have been set, which can be found in a recent review [38]. Furtherore projected sensitivities of Dark Matter direct detection experiments to effective WIMP-nucleus couplings have also appeared [39].

The above results combined with theoretical motivations stimulated interest in lower mass WIMPs, see e.g. the recent work 40]. In fact the first direct detection limits on sub-GeV dark matter from XENON10 have recently been obtained [41]. Subsequently detection of electrons in such searches has been considered [42]. It is encouraging that light WIMPs in the keV region can be detected employing Superfluid Helium [43].

It is, however, clear that lighter WIMPs, with a mass of the order of that of the electron, are quite different in energy and momentum transfer to the target. One, thus, needs suitable detectors, which maybe completely different from current WIMP detectors employed for heavy WIMP searches. In fact for WIMPs in the mass range of the electron mass the available energy is in the eV region and, thus, the detection of electron recoils is possible only for electrons with very low binding energies. Therefore the detector should be able to measure recoil energy in few eV region.

Regarding the evaluation of the elementary WIMP-electron cross section we will consider the following possibilities:

i) Scalar WIMPs. Such particles are viable cold dark matter candidates. Their mass, as far as we know, has not been constrained by any experiment. This scalar WIMP couples with ordinary Higgs with a quartic coupling, which has been inferred by the LHC experiments. Thus the WIMP interacts with electrons via Higgs exchange with an amplitude proportional to the electron mass $m_{e}$. In this case one gets a large kinematic enhancement of the cross section by a factor $m_{e}^{2} / m_{\chi}^{2}$ and, thus, WIMPs lighter than the electron are favored. For WIMPs with such a small mass $m_{\chi}$, however, the energy transfer to the electron is not adequate to overcome the electron binding. So the target must consist of essentially free electrons. We will discuss the availability of such targets below.

ii) For heavier WIMPs with masses up to 50 times that of the electron we will consider a model with a fermion WIMP interacting with the ordinary matter via a Z-exchange. In this case some electrons with low binding energies can be ejected and detected by their recoils. This model, due to the axial coupling, leads to a spin interaction among electrons. So, once the target is immersed in a magnetic field, one can have, as we will see, a variety of $\Delta m_{s}= \pm 1$ atomic spin excitations, both within the same shell or between spin-orbit partners, which can easily be detected.

iii) For WIMPs with masses greater than 50 times that of the electron, the electron binding is no longer a problem and practically all electrons of the atom can be ejected. We will not discuss this situation in any detail.

The paper is organized as follows: In section II we discuss the particle model employed. In section III we study the detection of almost free electrons in special low temperature detectors, e.g. superconducting materials, which act as caloremeters. We will exploit the enhancement of the obtained rates due to the scalar nature of the WIMPs. In section [V] we discuss the effect of the electron binding on the expected rates in the case of experiments measuring electron recoils ${ }^{2}$ in the case of WIMPs with a mass higher than that of the electron. In section $\mathrm{V}$ we discuss the

\footnotetext{
${ }^{2}$ We will not concern ourselves here with recently proposed Aromatic Organic Targets [44], [45] or other two-dimensional targets like those considered previously, see e.g. [46], [47]. The latter type of detectors will be considered separately elsewhere [48].
} 
possibility of detecting light WIMPs via atomic excitations. This can occur via the spin induced atomic transitions with excitation energy much smaller than the electron binding energy.

\section{THE PARTICLE MODEL.}

We will consider two such models:

\section{A. Scalar WIMPs interacting with the Higgs particle via a quartic coupling.}

Scalar WIMP's can occur in particle models. Examples are i) In Kaluza-Klein theories for models involving universal extra dimensions (for applications to direct dark matter detection see, e.g., [49]). In such models the scalar WIMPs are characterized by ordinary couplings, but they are expected to be quite massive. ii) extremely light particles [50], which are not relevant to the ongoing WIMP searches iii) Scalar isodoublet particles such as those considered previously in various extensions of the standard model [51] to provide some explanation for neutrino mass. Such particles can be long lived, protected by a discrete symmetry, and it is claimed that they can be a light dark matter candidate relevant for searches in WIMP-nucleus scattering.

In this work we will consider a particle model containing a scalar particle, whose mass, to our knowledge, has not yet been constrained by any experiment. This particle, indicated by $\chi$, can be a dark matter candidate, interacting with the neutral component $\phi_{0}$ of the standard model Higgs scalar, see Eqs (10) and (2) below, via a quartic coupling [52 55], and more recently [56]. It communicates with ordinary matter via Higgs exchange, see Fig. 1, and it becomes relevant for WIMP searches involving electrons.

The interest in such a WIMP has recently been revived due to a new scenario of dark matter production in bounce cosmology [57, 58] in which the authors point out the possibility of using dark matter as a probe of a big bounce at the early stage of cosmic evolution. A model independent study of dark matter production in the contraction and expansion phases of the big bounce reveals a new venue for achieving the observed relic abundance in which dark matter was produced completely out of chemical equilibrium [56]. In this case, this alternative route of dark matter production in bounce cosmology, can be used to test the bounce cosmos hypothesis [56].

The process

$$
\phi_{0}+\phi_{0} \rightarrow \chi+\chi
$$

involving the scalar WIMP $\chi$ and the neutral component $\phi_{0}$ of the Higgs scalar $\phi$ proceeds via the quartic coupling of the Higgs potential as described by the Feynman diagram shown in Fig. 1. Assuming that the surviving component of the scalar field $\phi$ is the Higgs $h$ discovered at the LHC, one can write down the cross section for both hadrons and electrons. The hadronic case has been studied before [56] and it is only mentioned here for copletenes and to indicate the importance of the communication between matter and ordinary metter via Higgs exchange.

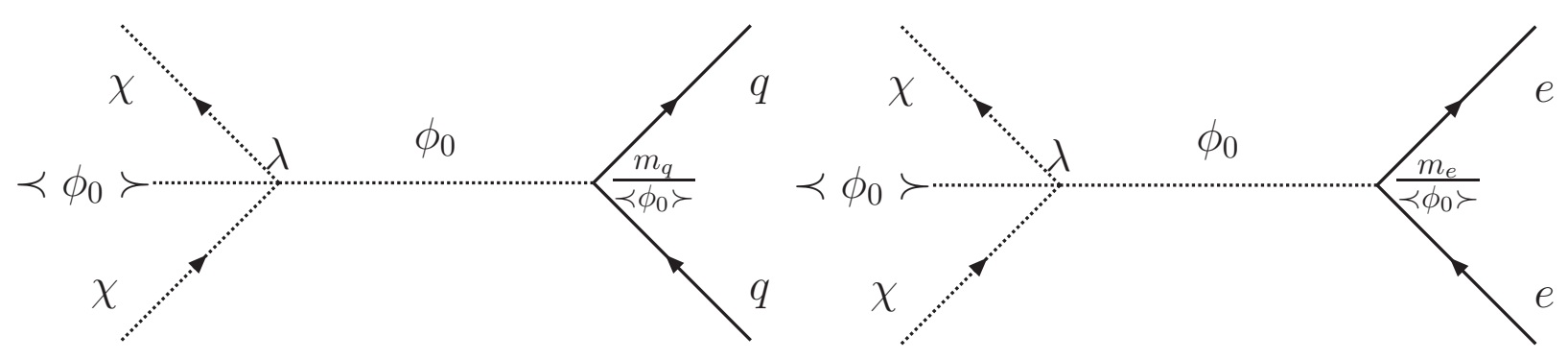

(a)

(b)

FIG. 1: (a) The quark - scalar WIMP scattering mediated by a scalar particle, i.e. via usual Higgs exchange $\phi_{0} \leftrightarrow h$.

(b) The corresponding diagram for electron scalar-WIMP scattering. Note that the relevant amplitudes are independent of the vacuum expectation value $\left\langle\phi_{0}\right\rangle$ of the scalar. 
In the case of the electron the elementary cross section is

$$
\sigma=\lambda^{2} \frac{1}{\left(2 m_{\chi}\right)^{2}} \frac{m_{e}^{2}}{m_{h}^{4}} \frac{1}{2 \pi} 2 \mu_{r}^{2}=\sigma_{0 H} \frac{1}{(1+x)^{2}} .
$$

with $m_{\chi}$ and $m_{h}$ being the masses of the scalar WIMP and the Higgs particle respectively, $\mu_{r}$ the reduced mass of the WIMP-electron system, $x=\frac{m_{\chi}}{m_{e}}$ and

$$
\sigma_{0 H}=\frac{1}{4 \pi} \lambda^{2} \frac{m_{e}^{2}}{m_{h}^{4}}=8.4 \times 10^{-45} \mathrm{~cm}^{2}=8.4 \times 10^{-9} \mathrm{pb}
$$

In deriving this scale of the cross section we have assumed that the quantity $\lambda$ is the same as the quartic coupling appearing in the Higgs potential. This is determined by the LHC data, $\lambda=1 / 2$. In the context of dark matter interactions it is a rather large cross section. It is the result of the fact that, in the small Yukawa coupling $f=\frac{m_{e}}{\left\langle\phi_{0}\right\rangle}$, the vacuum expectation value $\left\langle\phi_{0}\right\rangle$ is canceled by that appearing in the quartic coupling. We thus emphasize that the cross section does not suffer from the suppression expected in the decay $h \rightarrow e^{-} e^{+}$in which $f$ appears and, thus, it cannot be constrained by the LHC data. To the best of our knowledge it is not constrained by any other data.

\section{B. Fermion WIMPs interacting via Z-exchange.}

Such a mechanism has been considered in the case of the lightest supersymmetric particle (LSP) for the spin induced hadron cross section and more recently in the WIMP electron scattering [59]. The resulting cross section depends on the coupling of the dark neutral fermions to the Z-boson, i.e. it depends on the nature of the standard model (SM) fermion and the nature of the dark matter:

$$
\mathcal{L}=\frac{1}{2 \sqrt{2}} G_{F} J_{\mu}^{Z}(\chi) J^{Z \mu}(f)=\frac{1}{2 \sqrt{2}} G_{F} K_{\mu}\left(\bar{f} \gamma^{\mu}\left(g_{V}-g_{A} \gamma_{5}\right) f\right.
$$

In the above expression $K_{\mu}=g_{\chi}\left(\bar{\chi} \gamma_{\mu}\left(1-\gamma_{5}\right) \chi\right)$ stands for the axial coupling of the WIMP to the Z boson, analogous to $\mathrm{V}$-A of ordinary matter. We are interested in the axial current component, since the Fermi-like coupling of the electron vanishes. We will assume further that the strength of axial current is unity $g_{\chi}=g_{V}=g_{A}=1$. Then the invariant amplitude squared takes the form:

$$
\mathcal{M}^{2}=\frac{1}{8} G_{F}^{2} g_{A}^{2}
$$

Before proceeding further we will estimate the elementary WIMP-electron cross section for non relativistic electrons:

$$
d \sigma=\frac{1}{v} \frac{1}{8} G_{F}^{2} q^{2} d q d \xi \delta\left(q v \xi-\frac{q^{2}}{2 \mu_{r}}\right), \mu_{r}=\text { reduced mass of the WIMP electron system, }
$$

(see Eq. (11), section III for a kinematical derivation). Here $v$ is the velocity of the oncoming WIMP, $q$ is the momentum transfer to the electron and $\xi=\hat{v} \cdot \hat{q}$. This leads to the total cross section:

$$
\sigma_{e}=\frac{1}{8} G_{F}^{2} \frac{1}{\pi} \mu_{r}^{2}=\frac{1}{8} G_{F}^{2} \frac{1}{\pi} m_{e}^{2} \frac{x^{2}}{(1+x)^{2}}=\sigma_{0 Z} \frac{x^{2}}{(1+x)^{2}}
$$

with

$$
\sigma_{0 Z}=\frac{1}{8} G_{F}^{2} \frac{1}{\pi} m_{e}^{2} \approx 1.0 \times 10^{-9}
$$

It may be interesting to mention that one can infer the electron cross section from information on the corresponding nucleon cross section, which has been constrained by the WIMP-nucleus scattering for a WIMP mass, e.g. of 2 GeV, i.e. $\mu_{r}=\frac{2}{3} m_{p}$, by the CRESST-TUM40 experiment [60]. Such a phenomenological analysis is not, however, reliable, since the $\mu_{r}$ involved is much larger. In any case, it yields a cross section which is only a factor of three larger compared to that of Eq. 7 obtained theoretically. 
In this work, since $\sigma_{0 H}$ and $\sigma_{0 Z}$ do not differ much, for simplicity and to make easier a comparison of the dependence of the obtained results on other important features of the models, we will assume a common elementary cross section $\sigma_{0}$ for both Higgs and $Z$ exchange, which the average of the two.

$$
\sigma_{0 Z} \approx \sigma_{0 H}=\sigma_{0}=4.0 \times 10^{-45} \mathrm{~cm}^{2}=4.0 \times 10^{-9} \mathrm{pb}
$$

In any case this does not significantly affect the conclusions of the paper and, if necessary, one can re-scale the obtained rates.

\section{THE WIMP-ELECTRON RATE FOR FREE ELECTRONS}

The evaluation of the rate proceeds as in the case of the standard WIMP-nucleon scattering, but we will give the essential ingredients here to establish notation. We will begin by examining the case of a free electron.

i) The case of the scalar WIMPs $(\mathrm{SW})$ :

The differential cross section, when all particles involved are non relativistic and the initial electron is at rest, can be cast in the form:

$$
d \sigma=\frac{1}{v} \lambda^{2} \frac{1}{\left(2 m_{\chi}\right)} \frac{m_{e}^{2}}{m_{h}^{4}} \frac{1}{(2 \pi)^{2}} d^{3} \mathbf{p}_{\chi}^{\prime} d^{3} \mathbf{q} \delta\left(\mathbf{p}_{\chi}-\mathbf{p}_{\chi}^{\prime}-\mathbf{q}\right) \delta\left(\frac{\mathbf{p}_{\chi}^{2}}{2 m_{\chi}}-\frac{\mathbf{p}_{\chi}^{\prime 2}}{2 m_{\chi}}-\frac{\mathbf{q}^{2}}{2 m}\right)
$$

where $v$ is the velocity of the oncoming WIMP. The factor $1 /\left(2 m_{\chi}\right)^{2}$ is the usual normalization for the scalar particles and $m_{h} \approx 126 \mathrm{GeV}$ the mass of the exchanged Higgs particle. $\mathbf{p}_{\chi}, \mathbf{p}_{\chi}^{\prime}$ and $\mathbf{q}$ are the momenta of the oncoming and outgoing WIMP and the recoiling electron respectively. The last $\delta$ function expresses the energy conservation, since the participating particles are non relativistic. Integrating over the momenta we find:

$$
d \sigma=\frac{1}{2} \sigma_{0 H} \frac{1}{m_{\chi}^{2}} \frac{1}{v} q^{2} d q d \xi \delta\left(q v \xi-\frac{q^{2}}{2 \mu_{r}}\right), \mu_{r}=\frac{m_{e} m_{\chi}}{m_{e}+m_{\chi}}=\text { reduced mass }
$$

From the energy conserving $\delta$ function one finds that the momentum $\mathbf{q}$ transferred to the electron is given by

$$
q=2 m_{r} v, v=\text { WIMP velocity, } \xi=\hat{v} \cdot \hat{q} \geq 0
$$

Integrating over $\xi$ with the use of the delta function one finds :

$$
d \sigma=\sigma_{0} \frac{1}{v^{2}} \frac{1}{2 m_{\chi}^{2}} m_{e} d T=\sigma_{0} \frac{1}{2 v^{2}} \frac{1}{x^{2}} \frac{d T}{m_{e}}, x=\frac{m_{\chi}}{m_{e}}, \sigma_{0}=\sigma_{0 H},
$$

where $T$ is the kinetic energy of the outgoing electron given by:

$$
T=\frac{q^{2}}{2 m_{e}}=2 \frac{1}{m_{e}} \mu_{r}^{2} v^{2} \xi^{2}=2 m_{e} \frac{m_{\chi}^{2}}{m_{e}^{2}+m_{\chi}^{2}} v^{2} \xi^{2}=2 m_{e} v^{2} \xi^{2} \frac{x^{2}}{(1+x)^{2}}
$$

ii) The case of the fermion WIMP (FW).

Proceeding as above we find

$$
d \sigma=\sigma_{0 Z} \frac{1}{2 v^{2}} \frac{d T}{m_{e}}
$$

We are now going to discuss some parameters, which depend only on the mass of the WIMP and the velocity distribution. These are the maximum and the average electron energy. Their knowledge provides a qualitative understanding of the results expected from the detailed calculation.

From Eq. (12) we find that the fraction of the energy of the WIMP transferred to the electron is

$$
\frac{T}{K_{\chi}}=\xi^{2} \frac{x}{(1+x)^{2}}, x=\frac{m_{\chi}}{m_{e}},
$$


where $K_{\chi}=\frac{1}{2} m_{\chi} v^{2}=\frac{1}{2} m_{e} v^{2} x$ is the kinetic energy of the oncoming WIMP. We see that the maximum fraction occurs when $x=1$.

The maximum energy transfer is

$$
T_{\max }=2 m_{e} v_{\text {esc }}^{2} \frac{x^{2}}{(1+x)^{2}}
$$

i.e., in addition to $x$, it depends on the escape velocity, which is assumed to be $v_{e s c} \approx 2.84 v_{0}$ with $v_{0}=0.710^{-3} c$ the Sun's velocity round the center of the galaxy.

The electron recoiling energy depends on the direction of recoil. Its average over all directions is $\langle T\rangle_{r}=\frac{2}{3} v^{2} \frac{x^{2}}{(1+x)^{2}}$. Folding this with the velocity distribution, normally assumed to be of the form given by Eq. (40) with an upper cut off equal to $v_{e s c}$, we obtain the average energy transfer $\langle T\rangle_{r v}$, which depends on $x$. The maximum and the average energy transfers $T_{\max }$ and $\langle T\rangle_{r v}$ respectively are exhibited in fig. 2. This explains why for WIMP mass in the MeV

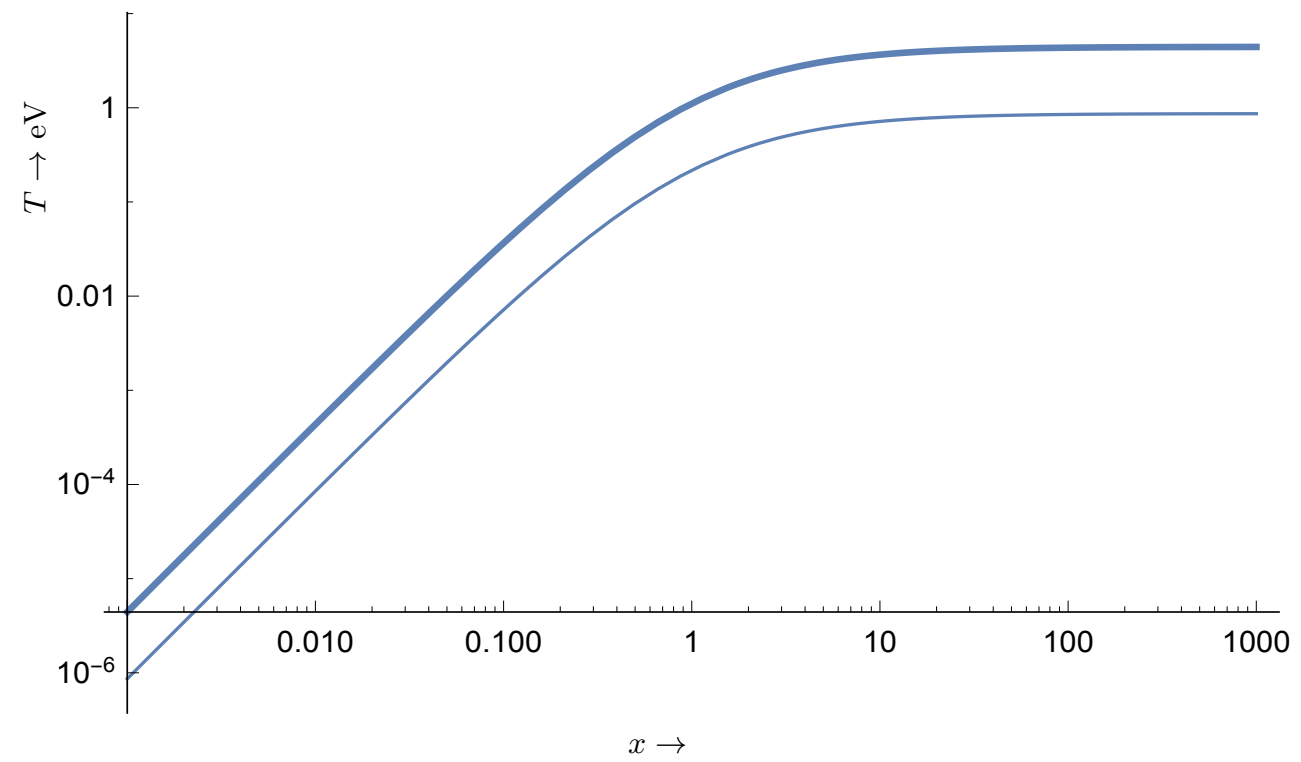

FIG. 2: The maximum (thick solid line) and the average (fine solid line) energy transfer in $\mathrm{eV}$ as a function of $x=\frac{m_{\chi}}{m_{e}}$ in the case of a free electron.

region the average energy transfer is in the range of a fraction of $\mathrm{eV}$, which is not perhaps so surprising, since, as we mentioned in the introduction, in the earlier hadronic WIMP searches, GeV WIMP masses implied an energy transfer in the keV region. The average energy can also be obtained by convoluting the energy transfer with the differential rate (for more details see [59]). Knowledge the average energy is also useful in coloremetric detectors. The maximum energy affects, of course, the expected total event rate.

Furthermore for a given energy transfer $T$ we find:

$$
v=\sqrt{\frac{m_{e} T}{2 \mu_{r}^{2} \xi^{2}}}=\left(1+\frac{1}{x}\right) \sqrt{\frac{1}{2} \frac{T}{m_{e}}} \rightarrow v \geq\left(1+\frac{1}{x}\right) \sqrt{\frac{1}{2} \frac{T}{m_{e}}} \rightarrow v_{\min }=\left(1+\frac{1}{x}\right) \sqrt{\frac{1}{2} \frac{T}{m_{e}}} .
$$

In other words the minimum velocity consistent with the energy transfer $T$ and the WIMP mass is constrained as above. The maximum velocity allowed is determined by the velocity distribution and it will be indicated by $v_{e s c}$. From this we can obtain the differential rate per electron in a given velocity volume $v^{2} d v d \Omega$ as follows:

$$
d R=\sigma_{0} \frac{\rho_{\chi}}{m_{\chi}} \frac{1}{2} v \nu(x) \frac{d T}{m_{e}} f(\boldsymbol{v}) d v d \Omega, \nu(x)=\left\{\begin{array}{cc}
x^{2}, & \text { sw } \\
1, & \text { FW }
\end{array}\right.
$$

where $f(\boldsymbol{v})$ is the velocity distribution of WIMPs in the laboratory frame. Integrating over the allowed velocity distributions we obtain:

$$
d R=\frac{\rho_{\chi}}{m_{\chi}} \sigma_{0} \frac{d T}{m_{e}} \frac{1}{2 v_{0}} \eta\left(v_{\min }\right) \times\left\{\begin{array}{cc}
x^{2}, & \text { sw } \\
1, & \text { FW }
\end{array}, \eta\left(v_{\min }\right)=\int_{v_{\min }}^{v_{e s c}} f(\boldsymbol{v}) v d v d \Omega\right.
$$


The parameter $\eta\left(v_{\min }\right)$ is a crucial parameter.

Before proceeding further we find it convenient to express the velocities in units of the Sun's velocity. We should also take note of the fact the velocity distribution is given with respect to the center of the galaxy. For a M-B distribution this takes the form:

$$
f\left(y^{\prime}\right)=\frac{1}{\pi \sqrt{\pi}} e^{-y^{\prime 2}}, y^{\prime}=\frac{v^{\prime}}{v_{0}}, v_{0}=220 \mathrm{~km} / \mathrm{s}
$$

We must transform it to the local coordinate system :

$$
\mathbf{y}^{\prime} \rightarrow \mathbf{y}+\hat{v}_{s}+\delta\left(\sin \alpha \hat{x}-\cos \alpha \cos \gamma \hat{y}+\cos \alpha \sin \gamma \hat{v}_{s}\right), \delta=\frac{v_{E}}{v_{0}}
$$

with $\gamma \approx \pi / 6, \hat{v}_{s}$ a unit vector in the Sun's direction of motion, $\hat{x}$ a unit vector radially out of the galaxy in our position and $\hat{y}=\hat{v}_{s} \times \hat{x}$. The last term, in parenthesis, in Eq. (19) corresponds to the motion of the Earth around the Sun with $v_{E} \approx 28 \mathrm{~km} / \mathrm{s}$ being the modulus of the Earth's velocity around the Sun and $\alpha$ the phase of the Earth ( $\alpha=0$ around June third). The above formula assumes that the motion of both the Sun around the Galaxy and of the Earth around the Sun are uniformly circular. The last term in Eq. (19) containing $\delta$ is vey important in estimating the modulation effect, i.e. the time dependence of the rate. Since $\delta$ is small we can expand the distribution in powers of $\delta$ keeping terms up to linear in $\delta$.

$$
d R=\left(\frac{\rho_{\chi}}{m_{\chi}} v_{0}\right) N_{e} \frac{1}{2 v_{0}^{2}} \frac{d T}{m_{e}}\left(\Psi_{0}\left(y_{\min }\right)+\Psi_{1}\left(y_{\min }\right) \cos \alpha\right) \times\left\{\begin{array}{cc}
x^{2}, & \mathrm{sw} \\
1, & \text { Fw }
\end{array}, x=\frac{m_{\chi}}{m_{e}},\right.
$$

where in the above equation the first term in parenthesis represents the average flux of WIMPs and the second term gives the number $N_{e}$ of electrons available for the scattering ${ }^{3}$. Furthermore .

$$
y_{\min }=\frac{v_{\min }}{v_{0}}=\frac{1}{v_{0}}\left(1+\frac{1}{x}\right) \sqrt{\frac{1}{2} \frac{T}{m_{e}}}, y_{e s c}=\frac{v_{e s c}}{v_{0}}
$$

For a M-B distribution one finds [59]:

$$
\Psi_{0}(x)=\frac{1}{2} H\left(y_{\text {esc }}-x\right)\left[\operatorname{erf}(1-x)+\operatorname{erf}(x+1)+\operatorname{erfc}\left(1-y_{\text {esc }}\right)+\operatorname{erfc}\left(y_{\text {esc }}+1\right)-2\right], x=y_{\min }
$$

and

$$
\begin{aligned}
\Psi_{1}(x)= & \frac{1}{2} H\left(y_{e s c}-x\right) \delta\left[\frac{-\operatorname{erf}(1-x)-\operatorname{erf}(x+1)-\operatorname{erfc}\left(1-y_{\mathrm{esc}}\right)-\operatorname{erfc}\left(y_{\mathrm{esc}}+1\right)}{2}\right. \\
& \left.+\frac{e^{-(x-1)^{2}}}{\sqrt{\pi}}+\frac{e^{-(x+1)^{2}}}{\sqrt{\pi}}-\frac{e^{-\left(y_{\mathrm{esc}}-1\right)^{2}}}{\sqrt{\pi}}-\frac{e^{-\left(y_{\mathrm{esc}}+1\right)^{2}}}{\sqrt{\pi}}+1\right], x=y_{\mathrm{min}}
\end{aligned}
$$

where $\operatorname{erf}(\mathrm{t})$ and $\operatorname{erfc}(\mathrm{t})$ are the well known error function and its complement respectively. In the above expression the Heaviside function $H$ guarantees that the required kinematical condition is satisfied.

After this formalism we are going to proceed in evaluating the expected spectrum of the recoiling electrons. The expression given by Eq. (20) can be cast in the form:

$$
\frac{d R}{d(T / 1 \mathrm{eV})}=\rho \Lambda\left(\Sigma_{0}\left(\frac{m_{\chi}}{m_{e}}, \frac{T}{(1 \mathrm{eV})}\right)+\Sigma_{1}\left(\frac{m_{\chi}}{m_{e}}, \frac{T}{(1 \mathrm{eV})}\right) \cos \alpha\right), \rho=\frac{1 \mathrm{eV}}{2 m_{e} v_{0}^{2}} \approx 2
$$

where

$$
\Sigma_{i}(x, s)=\frac{1}{x} \Psi_{i}\left(1.23\left(1+\frac{1}{x}\right) \sqrt{\rho s}\right) \times\left\{\begin{array}{ll}
\frac{1}{x^{2}}, & \text { sw } \\
1, & \mathrm{Fw}
\end{array}, i=0,1, s=\frac{T}{1 \mathrm{eV}}\right.
$$

\footnotetext{
${ }^{3}$ In standard targets $N_{e}=\frac{m_{t} Z_{e f f}}{A m_{p}}$, in a target of mass $m_{t}$ containing atoms with mass number $A, Z_{\text {eff }}$ represents the number of available electrons. The meaning of $Z_{e f f}$ becomes clear if one takes into account that the electrons are not free but bound in the atom see section IV Thus they are not all available for scattering, i.e. $Z_{\text {eff }}<<Z$.
} 
and

$$
\Lambda=\frac{\rho_{\chi}}{m_{e}} \sigma_{0} v_{0} N_{e}
$$

Where $N_{e}$ the number of electrons in the target.

The total event rates, assuming zero detector energy threshold, are given by:

$$
R_{i}=\Lambda \rho \int_{0}^{s_{\max }} d s \Sigma_{i}(x, s), s_{\max }=\frac{T_{\max }}{1 \mathrm{eV}} .
$$

The time average rate $R_{0}$ is exhibited in Fig. 3 a for a detector at zero anergy threshold.

In Fig 35 we show the effect of energy threshold on the rate by plotting the ratio of the rate at a thershold energy $\epsilon_{t h}$ divided by that at zero threshold, $R_{0}\left(\epsilon_{t h}\right) / R_{0}(0)$, as a function of $\epsilon_{t h}$. We prefer to show this ratio rather than the individual rate because it is independent of some parameters of the theory, e.g, the elementary electron cross section, whether the WIMP is a scalar or Fermion etc.

For the time dependence we prefer to present:

$$
R_{r}=\frac{R_{1}}{R_{0}} \cos \alpha, \alpha=\text { the phase of the Earth, }
$$

Where $R_{r}$ is essentially independent of $x$ and is exhibited in Fig. 3 b.

It is thus obvious for light WIMPs it is necessary to consider special materials in which the electrons are loosely bound, like electron pairs in a superconductor [61], provided, of course, that the number of these electrons is not very small. As another example we mention the recently proposed superconducting nanowires [62]. The latter has an energy threshold of $0.8 \mathrm{eV}$, whose effect on the rate will be discussed below

We will, therefore, estimate the rate for free electrons, i.e. estimate $\Lambda$ considering the following input:

- the elementary cross section $\sigma_{0}=4 \times 10^{-9} \mathrm{pb}=4 \times 10^{-45} \mathrm{~cm}^{2}$ both for the $\mathrm{Z}$ and Higgs exchange.

- The particle density of WIMPs in our vicinity:

$$
n=0.3 \times 10^{3}\left(\mathrm{MeV} / \mathrm{cm}^{3}\right) / 0.511 \mathrm{MeV} \approx 600 \mathrm{~cm}^{-3}
$$

(we use the electron mass in this estimate, since the correct mass dependence has been included through the extra factor of $1 / x$ in Eq. (24)). This value leads to a flux:

$$
\Phi_{0}=n \times 220 \mathrm{~km} / \mathrm{s}=1.3 \times 10^{10} \mathrm{~cm}^{-2} \mathrm{~s}^{-1}=4.2 \times 10^{17} \mathrm{~cm}^{-2} \mathrm{y}^{-1}
$$

- The number of electrons in the target, estimated to be

$$
N_{e}=10^{24}
$$

We thus using Eq. (25) we obtain

$$
\Lambda \approx 1.7 \times 10^{-3} \mathrm{y}^{-1}
$$

From Fig. [3a we find the time average rate at zero threshold as follows:

- $x=1 \Rightarrow$

$$
R_{0}=0.36 \times \Lambda=6.0 \times 10^{-4} \mathrm{y}^{-1}
$$

both for Fermion and scalar WIMPs. This is the maximum for Fermion WIMPs.

- For scalar WIMPs

$$
\begin{aligned}
& x=10^{-2} \Rightarrow R_{0}=1.2 \times 10^{2} \times \Lambda=0.2 \mathrm{y}^{-1} \\
& x=10^{-3} \Rightarrow R_{0}=1.2 \times 10^{3} \times \Lambda=2 \mathrm{y}^{-1}
\end{aligned}
$$




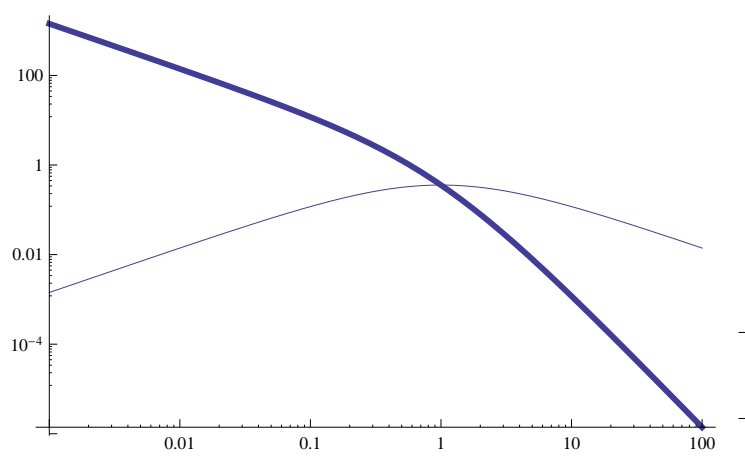

(a)

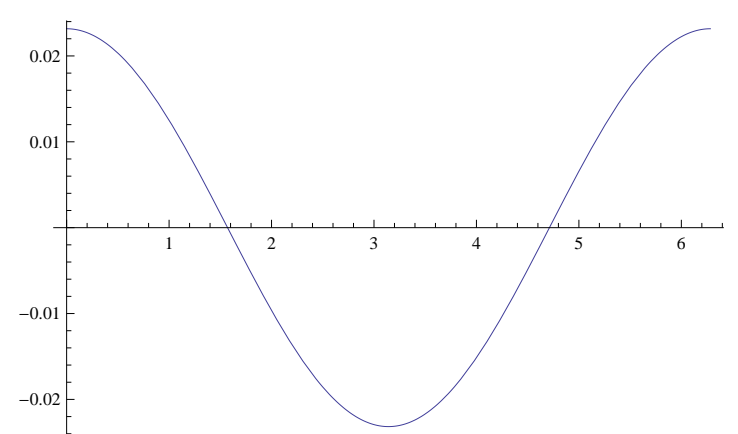

(b)

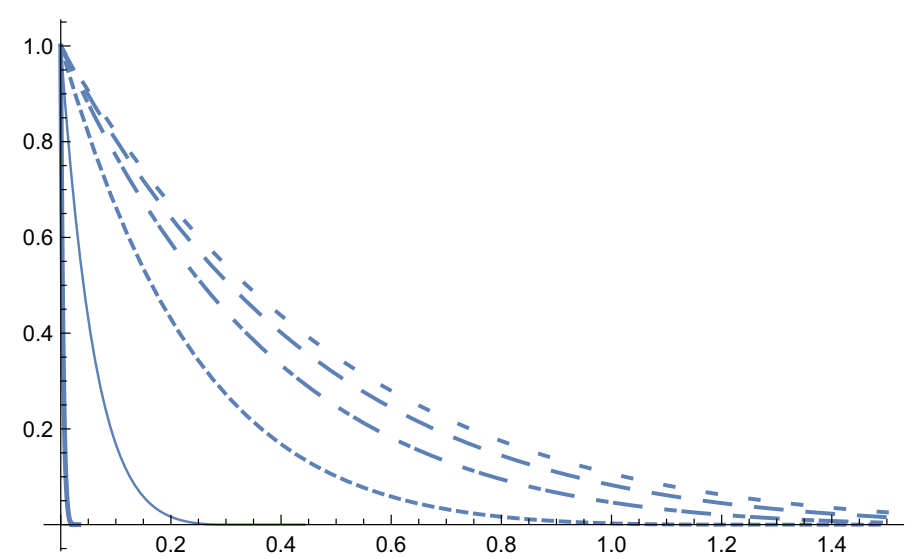

(c)

FIG. 3: (a) The total time averaged event rate $R_{0}$, in units of $\Lambda$, as a function of $x=\frac{m_{\chi}}{m_{e}}$. The thick and the fine solid lines correspond to a scalar and Fermion WIMP respectively.(b) The ratio of the time dependent to the time average rate, $\frac{R_{1}}{R_{0}} \cos \alpha$, as a function of the phase of the Earth $\alpha$ ( $\alpha=0$ around June third).(c) The ratio of the rate with a threshold energy $\epsilon_{t h}$ divided by that of zero theshold, $R_{0}\left(\epsilon_{t h}\right) / R_{0}(0)$, as a function $\epsilon_{t h}$ in eV. This ratio is the same for both scalar and Fermion WIMPs. The curves correspond to values of $x=0.5,1,2,5,10$ and 20 from left to right.

We should correct these values to take into account energy threshold effects of the detector, if necessary, according to Fig 3 .

We should mention, however, that the WIMP detection in calorimetric experiments is still difficult, since, in spite of the large rate in the case of scalar WIMPs, the total amount energy deposited in the detector for such a light WIMP is very small. Another important issue in the case of light WIMPs is the energy threshold of the detector. From Fig 3 . we see that the threshold of $0.8 \mathrm{eV}$ encountered in the proposed experiment with superconductor nanowires [62] can be overcome, even for small $x$, in particular for $x \geq 2$. The presence of threshold leads, of course, to a reduction of the expected rates.

Anyway it is encouraging that it seems possible, as it has recently been suggested in [61], to detect even very light WIMPS, much lighter than the electron, utilizing Fermi-degenerate materials like superconductors at low temperatures. In this case the energy required is essentially the gap energy of about $1.5 k T_{c}$ which is in the meV region, i.e the electrons are essentially free. These authors claim that in spite of the small energy in the range of few meV deposited to the system, the detection of very light WIMPs becomes feasible. Furthermore it has recently been proposed [63] that diamond targets can be sensitive to both absorption processes as well as electron recoils from dark matter scattering in the WIMP mass range of a few $\mathrm{MeV}$. 
TABLE I: The $\ell=0$ bound electron wave functions in momentum space. $a=\frac{\alpha Z}{n_{r}+\ell+1} \frac{m_{e} c^{2}}{\hbar c}, \alpha \approx \frac{1}{137}$.

\begin{tabular}{|c|c|}
\hline$n_{r}$ & $\Phi_{n r}(a, q)$ \\
\hline 0 & $\frac{2 \sqrt{2} a^{5 / 2}}{\pi\left(a^{2}+q^{2}\right)^{2}}$ \\
1 & $\frac{4 \sqrt{2} a^{5 / 2}\left(q^{2}-a^{2}\right)}{\pi\left(a^{2}+q^{2}\right)^{3}}$ \\
2 & $\frac{2 \sqrt{2} a^{5 / 2}\left(3 a^{5}-10 a^{3} q^{2}+3 a q^{4}\right)}{\pi\left(a^{2}+q^{2}\right)^{4}}$ \\
3 & $\frac{8 \sqrt{2} a^{5 / 2}\left(-a^{6}+7 a^{4} q^{2}-7 a^{2} q^{4}+q^{6}\right)}{\pi\left(a^{2}+q^{2}\right)^{5}}$ \\
4 & $\frac{2 \sqrt{2} a^{5 / 2}\left(5 a^{4}-10 a^{2} q^{2}+q^{4}\right)\left(a^{4}-10 a^{2} q^{2}+5 q^{4}\right)}{\pi\left(a^{2}+q^{2}\right)^{6}}$ \\
5 & $\frac{4 \sqrt{2} a^{5 / 2}\left(-3 a^{10}+55 a^{8} q^{2}-198 a^{6} q^{4}+198 a^{4} q^{6}-55 a^{2} q^{8}+3 q^{10}\right)}{\pi\left(a^{2}+q^{2}\right)^{7}}$ \\
\hline
\end{tabular}

\section{THE WIMP-ELECTRON RATE FOR BOUND ELECTRONS}

In the presence of bound electrons the WIMP mass must be quite a bit larger than the mass of the electron, $x=\frac{m_{\chi}}{m_{e}}>1$. In this case it is advantageous to consider the $Z$-exchange. Thus the differential cross section for bound electrons ${ }^{4}$ takes the form:

$$
d \sigma=\frac{\pi}{m_{e}^{2}} \frac{1}{v} \sigma_{0 Z}|\mathcal{M}(\mathbf{q})|^{2} \frac{d^{3} \mathbf{q}}{(2 \pi)^{3}} \frac{d^{3} \mathbf{p}_{\chi}^{\prime}}{(2 \pi)^{3}} \frac{d^{3} \mathbf{p}_{A}}{(2 \pi)^{3}}(2 \pi)^{3} \delta\left(\mathbf{p}_{\chi}-\mathbf{p}_{\chi}^{\prime}-\mathbf{q}-\mathbf{p}_{A}\right)(2 \pi) \delta\left(\frac{\mathbf{p}_{\chi}^{2}}{2 m_{\chi}}-\frac{\left(\mathbf{p}^{\prime}\right)_{\chi}^{2}}{2 m_{\chi}}-\frac{q^{2}}{2 m_{e}}\right)
$$

where again $\mathbf{p}_{\chi}, \mathbf{p}_{\chi}^{\prime}$ are the momenta of the oncoming and outgoing WIMPs with mass $m_{\chi}$ and $v$ is the velocity of the oncoming WIMP. $\mathbf{q}$ and $\mathbf{p}_{A}$ are the momentum transfer to the electron and the atom respectively. The energy transfer to the atom does not appear in the energy conserving $\delta$ function, since it is negligible. Furthermore

$$
\mathcal{M}(\mathbf{q})=\int d \mathbf{r} e^{i \mathbf{q} \cdot \mathbf{r}} \psi_{n_{r}, \ell, m}(\mathbf{r})
$$

with $\psi_{n_{r}, \ell, m}(\mathbf{r})$ the bound electron wave function coordinate space. $\mathcal{M}(\mathbf{q})$ essentially represents the overlap between the electron bound wave function and the plane wave of the outgoing electron with momentum $\mathbf{q}$. It can be written as $(2 \pi)^{3 / 2} \Phi_{n_{r}, \ell, m}(a, \mathbf{q})$, with $\Phi_{n_{r}, \ell, m}(a, \mathbf{q})$ the bound electron wave function in momentum space with $a=\frac{\alpha Z}{n_{r}+\ell+1} \frac{m_{e} c^{2}}{\hbar c}$. For $\ell=0$ (s-states), which are of interest in the present work, they appear in table $\prod$ as $\Phi_{n r}(a, q)$.

Thus integrating over $\mathbf{p}_{A}$ with the help of the momentum conserving $\delta$ function we obtain

$$
d \sigma=\frac{\pi}{v} \frac{\sigma_{0 Z}}{m_{e}^{2}} \frac{1}{(2 \pi)^{2}} \Phi_{n_{r}, \ell}^{2}(a, \mathbf{q}) d^{3} \mathbf{p}_{\chi}^{\prime} d^{3} \mathbf{q} \delta\left(\frac{\mathbf{p}_{\chi}^{2}}{2 m_{\chi}}-\frac{\left(\mathbf{p}^{\prime}\right)_{\chi}^{2}}{2 m_{\chi}}-\frac{\mathbf{q}^{2}}{2 m_{e}}\right)
$$

Then

$$
\int d^{3} \mathbf{p}_{\chi}^{\prime} \delta\left(\frac{\mathbf{p}_{\chi}^{2}}{2 m_{\chi}}-\frac{\left(\mathbf{p}^{\prime}\right)_{\chi}^{2}}{2 m_{\chi}}-\frac{q^{2}}{2 m_{e}}\right)=4 \pi m_{\chi}^{2} v \sqrt{1-\frac{2(b+T}{m_{x} v^{2}}}
$$

4 Sometimes the expression is written involving $\frac{\sigma_{e}}{\mu_{r}^{2}}$ As we have seen in section $\amalg$ however,

Thus

$$
\sigma_{e}=\sigma_{0 Z} \frac{x^{2}}{(1+x)^{2}}
$$

$$
\frac{\sigma_{e}}{\mu_{r}^{2}}=\frac{\sigma_{0 Z}}{m_{e}^{2}}
$$

The reduced mass expression is preferred, if the WIMP-electron cross section is extracted phenomenologically. 
where $b$ is the binding energy of the electron and $T$ is the energy of the recoiling electron, $T=q^{2} /\left(2 m_{e}\right)$. Similarly the integration over $\mathbf{q}$ for s-wave functions yields $\Phi_{n_{r}, \ell}^{2}\left(a, \sqrt{2 m_{e} T}\right) 4 \pi \sqrt{2 m_{e} T} m_{e} d T$. Furthermore by writing $\sqrt{2 m_{e} T}=u a$ we get

$$
\Phi_{n_{r}, \ell}^{2}\left(a, \sqrt{2 m_{e} T}\right)=\frac{\psi_{n_{r}, \ell}^{2}(u)}{a^{3}}
$$

Thus the cross section becomes

$$
d \sigma=\frac{4 \pi}{y} \sigma_{0 Z} x^{2} \psi_{n_{r}, \ell}^{2}(u) u \sqrt{y^{2}-\frac{2(b+T}{x m_{e} v_{0}^{2}}} \frac{m_{e} d T}{a^{2}}, y=\frac{v}{v_{0}}
$$

where having in mind to eventually use the Maxwell-Boltzmann (M-B) velocity distribution we have expressed the velocity in units of $v_{0}=220 \mathrm{~km} / \mathrm{s}$. Measuring now the $b$ and $T$ in $\mathrm{eV}$, which is the expected scale, we obtain

$$
d \sigma=\frac{4 \pi}{y} \sigma_{0 Z} x^{2} \psi_{n_{r}, \ell}^{2}(u(T)) \sqrt{y^{2}-\frac{\rho^{\prime}(b+T)}{x}}\left(\frac{n_{r}+\ell+1}{\alpha Z}\right)^{2} \times 2 \times 10^{-6} u(T) d T, \rho^{\prime}=3.64
$$

where

$$
u(T)=\sqrt{\frac{0.2}{m_{e}}} \frac{n_{r}+\ell+1}{\alpha Z} \sqrt{T} \approx 6.3 \times 10^{-4} \frac{n_{r}+\ell+1}{\alpha Z} \sqrt{T}
$$

The behavior of the function $\psi_{n_{r}, \ell}^{2}(u(T))$ for $\alpha Z \approx \frac{1}{2}$ for various values of $n_{r}$ is exhibited in Fig. 4 One can see that the higher $n_{r}$ are favored. For a given $n_{r}$ it is essentially independent of $T$ for recoiling energies of interest to us.

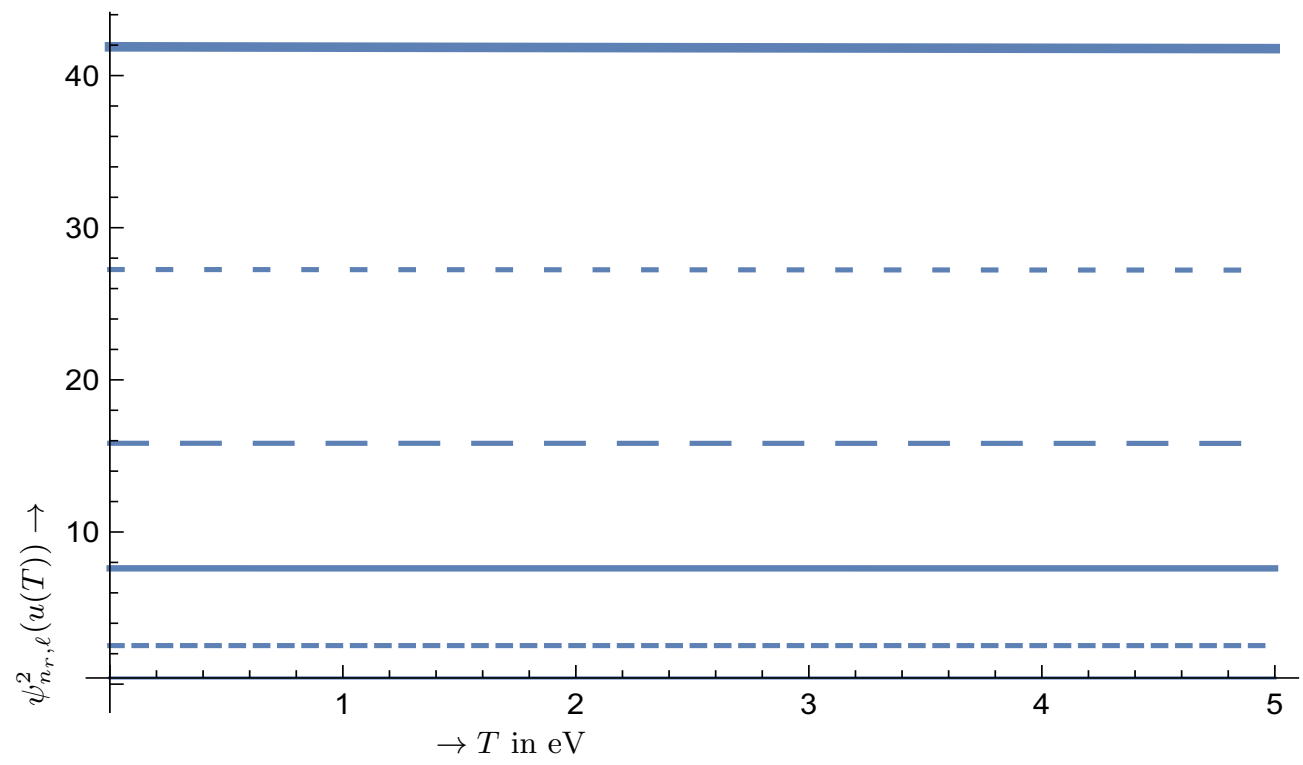

FIG. 4: The function $\psi_{n_{r}, \ell}^{2}(u(T))$ for $\alpha Z=1 / 2$ is exhibited as a function of the electron recoil energy $T$ in units of $\mathrm{eV}$. It is shown for $n_{r}=0,1,2,3,4,5$ increasing upwards (the lowest one is barely visible).

Returning now to Eq. (31) we notice:

i) in folding with the velocity distribution we must integrate between $y_{\min }=\sqrt{\frac{2 \rho^{\prime}(b+T)}{x}}$ and $y_{\text {esc }}=2.84$

ii) for a given $x$ and $b$ the maximum electron energy is

$$
\frac{T_{\max }}{1 \mathrm{ev}}=\frac{y_{e s c}^{2} x}{2 \rho^{\prime}}-\frac{b}{1 \mathrm{ev}}=1.1 x-\frac{b}{1 \mathrm{ev}}
$$

Thus for a value of $x=5$ and a binding energy $2.5 \mathrm{eV}$ the maximum electron energy is expected to be $3 \mathrm{eV}$. 
TABLE II: Listed are the atoms and the binding energy of the corresponding s-electrons. Only electrons with binding energies less than $10 \mathrm{eV}$ are listed.

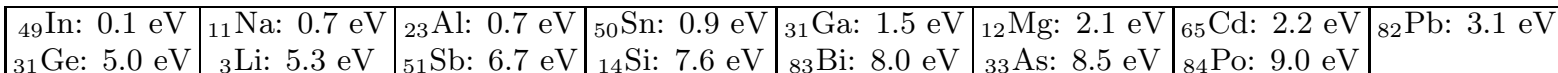

iii) For a given binding energy, $x$ must be at least $x_{\min }=0.90 b$

Folding the cross section with the velocity distribution (see Eq. (40) below) including the extra factor of $y$ coming from the flux we obtain:

$$
\begin{aligned}
\left\langle y \frac{d \sigma}{d T}\right\rangle & =4 \pi \sigma_{0 Z} x^{2} \psi_{n_{r}, \ell}^{2}\left(\frac{n_{r}+\ell+1}{\alpha Z}\right)^{2} \times 2 \times 10^{-6} u(T) d T g(x, T, b), \\
g(x, T, b) & =\frac{2}{\sqrt{\pi}} \int_{y_{\text {min }}}^{y e s c} d y y e^{-\left(1+y^{2}\right)} \sinh 2 y \sqrt{y^{2}-\frac{\rho^{\prime}(b+T)}{x}}
\end{aligned}
$$

The total rate can now be cast in the form

$$
\begin{gathered}
\frac{d R}{d T}=\Lambda R_{d_{0}}, R_{d_{0}}=4 \pi x \psi_{n_{r}, \ell}^{2}\left(\frac{n_{r}+\ell+1}{\alpha Z}\right)^{2} \times 2 \times 10^{-6} u(T) g(x, T, b) \\
R=\Lambda R_{0}, R_{0}=4 \pi x \int_{0}^{T \max (x, b)} d T \psi_{n_{r}, \ell}^{2}\left(\frac{n_{r}+\ell+1}{\alpha Z}\right)^{2} \times 2 \times 10^{-6} u(T) g(x, T, b)
\end{gathered}
$$

where

$$
\Lambda=\frac{\rho_{\chi}}{m_{e}} \sigma_{0 Z} v_{0}
$$

with $\rho_{\chi}$ the WIMP density in our vicinity. Note that $m_{e}$ rather $m_{\chi}$ has been employed in determining the number density of WIMPs with a compensating factor $1 / x$ already incorporated into Eq. (35).

There exist few atoms which possess s-state electrons with small binding energies. From atomic data tables 64 66 we found and list those with $b \leq 10 \mathrm{eV}$ in table [I] There exist, of course, states with binding energies smaller than those of the s-states, but, as we have mentioned, for light WIMPs they are not going to contribute significantly to the total rate. It thus appears that i) $\mathrm{NaI}(\mathrm{b}=0.7 \mathrm{eV}$ in Na) as scintillator and ii) $\mathrm{CdTe}(\mathrm{b}=2.2 \mathrm{eV}$ in $\mathrm{Cd}), \mathrm{Ge}(\mathrm{Li})(\mathrm{b}=5$ $\mathrm{eV}$ in $\mathrm{Ge}$ and $\mathrm{Li})$ and $\mathrm{Si}(\mathrm{b}=7.6 \mathrm{eV})$ can be used as solid state detectors.

Many of the elements listed in table II involving s-electrons with low binding energies can serve as good targets, provided, of course, that recoiling electrons with energies in the few $\mathrm{eV}$ can be detected. Once a special target is selected, one must make an orbit by orbit calculation, based on the data of table III and sum the cross section over all orbits multiplied with the number of electrons involved.

At this point we will make a simple calculation using $N_{e}=N_{A}=10^{25}$, which corresponds to the number of atoms of a $\mathrm{Kg}$ of an $A=60$ target. This is an order of magnitude larger than that used in the case of free electrons discussed in the previous section. We thus obtain the results shown in Fig. [5] using $Z_{\text {eff }}$ much smaller than $Z$ for a typical atom. In spite of the larger $N_{e}$, for low $x$ the obtained results are smaller than those obtained in the previous section. We can trace this suppression to the electron binding energy $b$ through atomic parameter $a$, which is of the order of $m_{e}$, much larger than the electron recoiling energies, which, for $x<10$, tend to be in the few $\mathrm{eV}$ region.

The results, of course, tend to further increase approximately linearly with $x$ and eventually, for $x>50$, electron recoils become easily detectable. For such values of $x$, of course, all electrons can participate, i.e. $Z_{\text {eff }}=Z$

\section{ATOMIC EXCITATIONS}

We have seen that detecting low mass WIMPs by observing recoiling electrons is pretty hard, since few electrons can be ejected, due to their binding in the atom. This problem does not persist, if the electrons are not ejected, but 


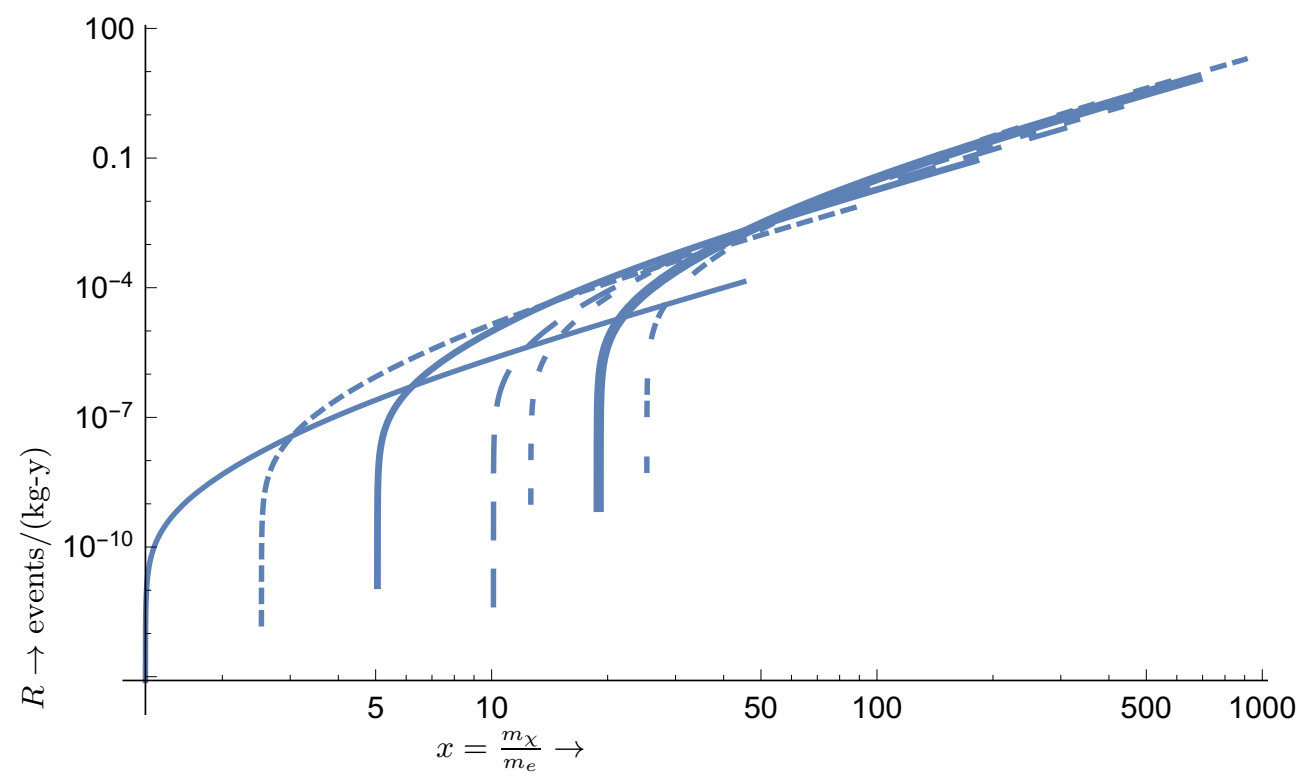

FIG. 5: The event rates as a function of $x$ for various electron binding energies $b$ in $\mathrm{eV}$, which are increasing from left to right. Thus the fine solid curve corresponds to $b=1\left(Z_{\text {eff }}=1.0\right)$, the short dashed curve $b=2\left(Z_{\text {eff }}=1\right)$, the intermediate thick solid line to $b=3\left(Z_{\text {eff }}=2\right)$ the long dashed curve $b=4\left(Z_{\text {eff }}=3\right)$, the intermediate short dashed curve $b=6\left(Z_{\text {eff }}=4\right)$, the very thick solid line to $b=10\left(Z_{\text {eff }}=6\right)$ and the short-long dashed curve to $\mathrm{b}=15\left(Z_{\text {eff }}=8\right)$. One can clearly see the threshold values of $x$ for a given binding energy $b$. For illustration purposes the hydrogenic wave function with $n_{r}=4, \ell=0$ has been employed.

promoted to a higher level and the de-excitation photons are observed. In this case an energy difference even much smaller than $\mathrm{eV}$ is available, if the target is placed in a magnetic field at low temperature.

As a matter of fact the axial current present in the Z-mediated WIMP-electron interaction through the electron spin can cause atomic transitions between atomic levels within states, which have the same radial quantum numbers and angular quantum numbers $j_{1}, m_{1}$ and $j_{2}, m_{2}$. If the atom is placed in a magnetic field the transition matrix element is expressed in terms of the Glebsch-Gordan coefficient and the nine- $\mathrm{j}$ symbol:

$$
\begin{aligned}
\mathcal{M}\left[\left(n, \ell, j_{1} m_{1}\right) \rightarrow\left(n, \ell, j_{2} m_{2}\right)\right] & =C_{\ell, j_{1}, m_{1}, j_{2}, m_{2}}, \\
C_{\ell, j_{1}, m_{1}, j_{2}, m_{2}} & =\left\langle j_{1} m_{1}, 1 m_{2}-m_{1} \mid j_{2} m_{2}\right\rangle \sqrt{\left(2 j_{1}+1\right) 3} \sqrt{2 \ell+1} \sqrt{6}\left\{\begin{array}{ccc}
\ell & \frac{1}{2} & j_{1} \\
\ell & \frac{1}{2} & j_{2} \\
0 & 1 & 1
\end{array}\right\}
\end{aligned}
$$

When $j_{1}=j_{2}$ the two states are those arising from the splitting of the degeneracy due to the Zeeman effect with an energy difference $\delta E=E_{f}-E_{i}=$ a few $\mu \mathrm{eV}$. If $j_{1} \neq j_{2}$ the two levels correspond the spin orbit partners with energy differences in the $\mathrm{eV}$ region. For the readers convenience these matrix elements are tabulated for some cases of practical interest and are given in the Appendix, see section VIII

The differential cross section now takes the form:

$$
d \sigma=\frac{1}{v} \sigma_{0 Z} \frac{\pi}{m_{e}^{2}} \frac{1}{(2 \pi)^{2}}\left(C_{\ell, j_{1}, m_{1}, j_{2}, m_{2}}\right)^{2} d^{3} \mathbf{p}_{\chi}^{\prime} d^{3} \mathbf{q} \delta\left(\mathbf{p}_{\chi}-\mathbf{p}_{\chi}^{\prime}-\mathbf{q}\right) \delta\left(\frac{\mathbf{p}_{\chi}^{2}}{2 m_{\chi}}-\frac{\mathbf{p}_{\chi}^{\prime 2}}{2 m_{\chi}}-\delta E\right)
$$

where $\mathbf{q}$ the momentum transfer to the atom and $\delta E$ the excitation energy. The recoil energy of the atom is negligible. Integrating over the momentum $\mathbf{q}$ we find:

$$
d \sigma=\frac{1}{v} \sigma_{0 Z} \frac{\pi}{m_{e}^{2}} \frac{1}{(2 \pi)^{2}}\left(C_{\ell, j_{1}, m_{1}, j_{2}, m_{2}}\right)^{2} d^{3} \mathbf{p}_{\chi}^{\prime} \delta\left(\frac{\mathbf{p}_{\chi}^{2}}{2 m_{\chi}}-\frac{\mathbf{p}_{\chi}^{\prime 2}}{2 m_{\chi}}-\delta E\right) .
$$


Performing the remaining integration we get

$$
d \sigma=\left.\frac{1}{v} \sigma_{0 Z} \frac{\pi}{m_{e}^{2}} \frac{1}{(2 \pi)^{2}}\left(C_{\ell, j_{1}, m_{1}, j_{2}, m_{2}}\right)^{2} 4 \pi \frac{\mathbf{p}_{\chi}^{\prime 2}}{\mid p_{\chi}^{\prime} / m_{\chi}}\right|_{p_{\chi}^{\prime}=\sqrt{p_{\chi}^{2}-2 m_{\chi} \delta E}}=\frac{1}{v} \sigma_{0 Z} \frac{m_{\chi}^{2}}{m_{e}^{2}}\left(C_{\ell, j_{1}, m_{1}, j_{2}, m_{2}}\right)^{2} \sqrt{v^{2}-\frac{2 \delta E}{m_{\chi}}}
$$

We must now fold it with the velocity distribution in the local frame, ignoring the motion of the Earth around the Sun, i.e.

$$
f\left(v, v_{0}, \xi\right)=\frac{1}{v_{0}^{3} \pi \sqrt{\pi}} e^{-\frac{v^{2}+2 v v_{0} \xi+v_{0}^{2}}{v_{0}^{2}}}
$$

The integral over $\xi$ is done analytically to yield:

$$
\langle(\sigma y)\rangle=\sigma_{0 Z}\left(C_{\ell, j_{1}, m_{1}, j_{2}, m_{2}}\right)^{2} \frac{m_{\chi}^{2}}{m_{e}^{2}} \frac{4}{\sqrt{\pi}} \int_{b}^{y_{\max }} d y y y^{2} e^{-y^{2}-1} \frac{\sinh (2 y)}{2 y} \sqrt{1-\frac{b^{2}}{y^{2}}}, b=\sqrt{\frac{2 \delta E}{m_{\chi} v_{0}^{2}}}
$$

( $b$ here should not be confused with the electron binding energy) or

$$
\begin{aligned}
\langle(\sigma y)\rangle= & \sigma_{0 Z}\left(C_{\ell, j_{1}, m_{1}, j_{2}, m_{2}}\right)^{2} \\
& x^{2} \frac{2}{\sqrt{\pi}} \int_{b}^{y_{\max }} d y y^{2} e^{-y^{2}-1} \sinh (2 y) \sqrt{1-\frac{b^{2}}{y^{2}}}, b=\sqrt{\frac{2 \delta E}{x m_{e} v_{0}^{2}}}, x=\frac{m_{\chi}}{m_{e}}
\end{aligned}
$$

The last integral can only be done numerically.

The event rate, omitting the orbit dependent angular momentum coefficient $\left(C_{\ell, j_{1}, m_{1}, j_{2}, m_{2}}\right)^{2}$ takes the form:

$$
R=\frac{\Lambda}{x} x^{2} \frac{2}{\sqrt{\pi}} \int_{b}^{y_{\max }} d y y^{2} e^{-y^{2}-1} \sinh (2 y) \sqrt{1-\frac{b^{2}}{y^{2}}}, b=\sqrt{\frac{7.3(\delta E / 1 \mathrm{eV})}{x}}
$$

where $\Lambda$ is defined as

$$
\Lambda=\frac{\rho_{\chi}}{m_{e}} \sigma_{0} v_{0} N_{e}
$$

One can easily find that the constraint among the parameters is

$$
\sqrt{\frac{7.3(\delta E / 1 \mathrm{eV})}{x}}<2.84 \Rightarrow x>0.9 \frac{\delta E}{1 \mathrm{eV}}
$$

The extra factor of $1 / x$ in Eq. (43) comes from the fact that the value of $\Lambda$ employed has been evaluated with WIMP number density associated with a mass $m_{e}$, rather than $m_{\chi}$.

\section{A. Some general trends}

The obtained results are exhibited in Fig. 6, both for $\Lambda=1$ and $\lambda=1.7 \times 10^{-3}$, assuming one electron per atom.

The detection involves measuring the de-excitation of the populated level. It is also possible, following Sikivie's ideas [67] for axion detection, to concentrate [68] on the population of a preferred atomic level at low excitation provided that it is not otherwise occupied by electrons. Then, assuming that it becomes occupied due to the WIMP-electron interaction, employ a tunable laser to further excite the electrons to a preferred level. One can thus observe the de-excitation of this preferred level. This may require to cool system at very low temperatures and perhaps use a target, enriched with an impurity, if necessary, so that the system maintains an atomic structure at the necessary low temperature.

The obtained rates in Fig. 6 are in principle detectable. It should be noted, however, that the angular momentum factors $\left(C_{\ell, j_{1}, m_{1}, j_{2}, m_{2}}\right)^{2}$ have not been included. They can be easily incorporated, once a target and a specific excitation pattern are selected. These can be found in tables [II IV] of the Appendix, section VIII.

An additional advantage of the atomic experiments is the fact that targets with a number of electrons $N_{e}>10^{24}$ are feasible. 


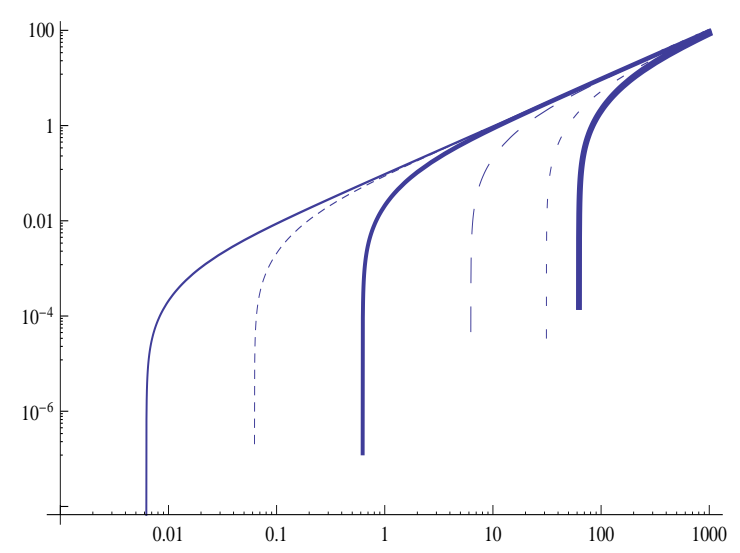

(a)

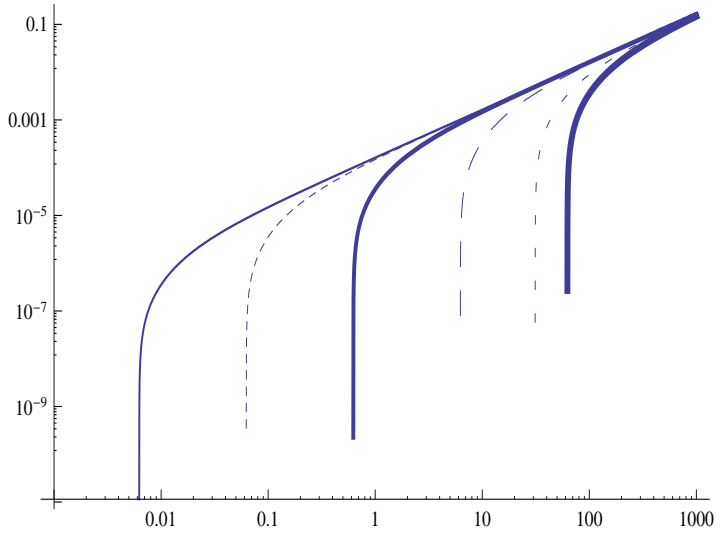

(b)

FIG. 6: The total event rate per year for a target with $N_{A}=10^{24}$ atoms as a function of $x=\frac{m_{x}}{m_{e}}$ in the case of atomic excitations. In panel (a) the various curves correspond to rates with $\Lambda=1$, while in panel (b) those with the actual value of $\Lambda=1.7 \times 10^{-3}$. In both cases the curves correspond to values of $\delta \mathrm{E}=\{0.001,0.01,0.1,1 ., 5 ., 10.\} \mathrm{eV}$ increasing from left to right.

\section{B. Some special targets}

We are going to examine some special examples.

i) First we will consider a target with the ground being a single $p_{1 / 2}$ orbital, while the $p_{3 / 2}$ is empty. Let us suppose that the spin orbit splitting is $\epsilon_{p}$. In the presence of a magnetic field the m-degeneracy is removed and the ground state is in the state $\left|j_{1}, m_{1}\right\rangle=|1 / 2,-1 / 2\rangle$. Then we have the following spin induced transitions:

$$
|1 / 2,-1 / 2\rangle \rightarrow|1 / 2,1 / 2\rangle,|1 / 2,-1 / 2\rangle \rightarrow|3 / 2,-3 / 2\rangle,|1 / 2,-1 / 2\rangle\rangle \rightarrow|3 / 2,-1 / 2\rangle,|1 / 2,-1 / 2\rangle \rightarrow|3 / 2,1 / 2\rangle
$$

indicated as A,B,C and D respectively. To leading order the spin $g_{s}$ factors are $g_{s}=(2 / 3,4 / 3)$ for $p_{1 / 2}$ and $p_{3 / 2}$ respectively. Thus the energies of the transitions are

$$
E_{x}=\left\{\frac{2 \delta}{3}, \epsilon_{p}-\frac{5 \delta}{3}, \epsilon_{p}-\frac{\delta}{3}, \delta+\epsilon_{p}\right\}
$$

where $\delta=\mu_{B} B$ with $\mu_{B}$ the Bohr magneton and $B$ the magnetic field. For a field of $1 \mathrm{~T}$ we find $\delta=5.788 \times 10^{-5} \mathrm{eV}$ A good candidate for such a transition is ${ }_{13} \mathrm{Al}$, involving the orbitals $2 p_{1 / 2}$ and $2 p_{3 / 2}$. We find $\epsilon_{p}=0.65$, which in good agreement with existing tables (https://www.nist.gov/pml/atomic-spectra-database). Thus

$$
E_{x}=\left\{0.0000386, \epsilon_{p}-0.0000965, \epsilon_{p}-0.0000193, \epsilon_{p}+0.0000579\right\}, C\{2 / 9,4 / 3,8 / 9,4 / 9\},
$$

where $C$ are the corresponding spin matrix elements.

ii) Next we will consider a target with the ground being containing a single $d_{3 / 2}$ orbital, while the $d_{5 / 2}$ is empty. Let us suppose that the spin orbit splitting is $\epsilon_{d}$. In the presence of a magnetic field the m-degeneracy is removed and the ground state is in the state $\left|j_{1}, m_{1}\right\rangle=|3 / 2,-3 / 2\rangle$. To leading order the $g_{s}$ values are $g_{s}=(4 / 5,6 / 5)$ for $d_{3 / 2}$ and $d_{5 / 2}$ Then we have the following spin induced transitions:

$$
|3 / 2,-3 / 2\rangle \rightarrow|3 / 2,1 / 2\rangle,|3 / 2,-3 / 2\rangle \rightarrow|5 / 2,-5 / 2\rangle,|3 / 2,-3 / 2\rangle \rightarrow|5 / 2,-3 / 2\rangle,|3 / 2,-3 / 2\rangle \rightarrow|5 / 2,-1 / 2\rangle
$$

indicated again as A,B,C and D respectively. Their energies are

$$
E_{x}=\left\{\frac{8 \delta}{5}, \epsilon_{d}-\frac{9 \delta}{5}, \epsilon_{d}-\frac{3 \delta}{5}, \epsilon_{d}+\frac{3 \delta}{5}\right\}
$$

Our best candidate found in the above reference is the target ${ }_{21} \mathrm{Sc}$ involving the $3 d 3 / 2 \rightarrow 3 d 5 / 2$ transitions with $\epsilon_{d}=0.021 \mathrm{eV}$. Other candidates can also be found in the same reference, e.g.: 
$\mathrm{Z}=39(\mathrm{Y} \mathrm{I}, 4 \mathrm{~d} 3 / 2,5 / 2,0.066 \mathrm{eV})$ and $\mathrm{Z}=71(\mathrm{Lu} \mathrm{I}, 5 \mathrm{~d} 3 / 2,5 / 2,0.25 \mathrm{eV}$, where I indicates that it is a neutral atom. We thus find

$$
E_{x}=\left\{0.000092608, \epsilon_{d}-0.000104184, \epsilon_{d}-0.000034728, \epsilon_{d}+0.000034728\right\}, C=\{4 / 25,8 / 5,16 / 25,4 / 25\}
$$

where again $C$ are the corresponding spin matrix elements.

iii) $s 1 / 2$ states. Such states exist in many atomic targets. In all such cases

$$
E_{x}=2 \delta=1.1 \times 10^{-3}, C=2 .
$$

We note the large spin matrix element.

We thus have to calculate for each target the rates

$$
R_{i}=C_{i} R[E x(i, x)], i=A, B, C, D,
$$

where $\mathrm{R}$ is the expression for the rate given above.

The obtained results are exhibited in 7 Note that in the case of $s 1 / 2$ and the A type transitions the thresh hold value of $x$, i.e. the lowest value of the WIMP mass required for the process to take place, is close to zero, since the spin orbit splitting does not appear. This also happens to be the case for all $3 \mathrm{~d}$-transitions considered here, since the spin orbit splitting is quite small $(0.021 \mathrm{eV})$. On the other hand in the case of 2 p-levels for the B,C,D transitions, a value of $m_{\chi} \geq 0.6 m_{e}$ is required, due to the fact that the spin orbit splitting is a bit higher $(0.65 \mathrm{eV})$.

We should not forget that the actual rates per year can be obtained after multiplying the rates exhibited in Fig. 7 with $\Lambda, \Lambda=1.7 \times 10^{-3}$ for $N=1 \times 10^{24}$ atoms.

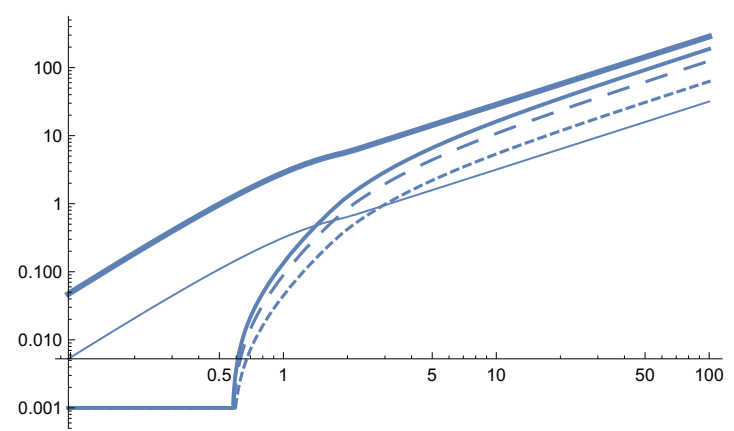

(a)

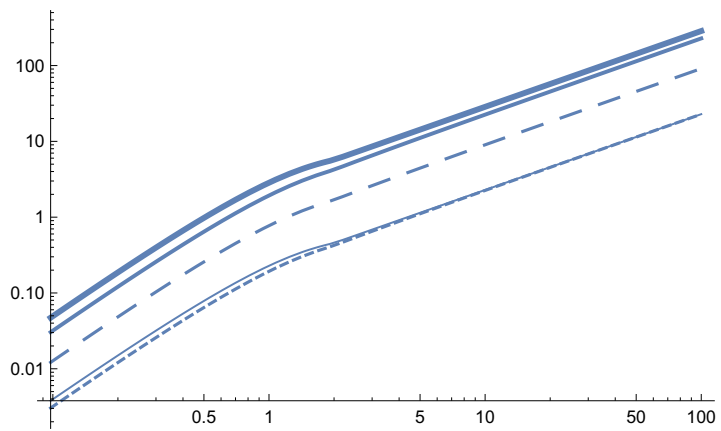

(b)

$$
\rightarrow x=\frac{m_{\chi}}{m_{e}}
$$

FIG. 7: The total event rates, obtained with $\Lambda=1$, for for the atomic excitations in the case of the special targets discussed in the text. (a) In the case $2 \mathrm{p} 1 / 2 \rightarrow 2 \mathrm{p} 3 / 2$ excitations in ${ }_{13} \mathrm{Al}$. (b) In the case of the $3 \mathrm{~d} 3 / 2 \rightarrow 3 \mathrm{~d} 5 / 2$ excitations in ${ }_{21} \mathrm{Sc}$. In both cases the solid line, thick solid line, long dash and short dash correspond to the excitations of type A,B,C and D respectively (see text). For comparison we present our results for the type of s1/2 excitations, indicated by the very thick solid line. Otherwise the notation is the same with that of fig. 6.

\section{COMPARISON OF ELECTRON RECOILS AND ATOMIC EXCITATIONS}

In the case of light WIMPs, it is of interest to compare the electron recoil rates with those of the atomic excitation experiments. The targets are not the same, but the number of atoms in the target is taken to be the same.

In the case of a target with free electrons we must compare Fig. 3 with Figs 6 and 7 The electron scattering for free electrons yields $0.36 \times 1.7 \times 10^{-3}=6.0 \times 10^{-4}$ events per year $\left(\mathrm{y}^{-1}\right)$, while those associated with $3 \mathrm{~d}$ transitions, also in $\mathrm{y}^{-1}$, are:

$$
x=1 \leftrightarrow\{0.000388372,0.00388372,0.00155349,0.000388372\},
$$




$$
\begin{gathered}
x=2 \leftrightarrow\{0.000776783,0.00776783,0.00310713,0.000776783\}, \\
x=5 \leftrightarrow\{0.00194202,0.0188501,0.00753977,0.00188488\}, \\
x=10 \leftrightarrow\{0.00388407,0.0382793,0.0153115,0.00382781\}, \\
x=50 \leftrightarrow\{0.0194205,0.193653,0.0774611,0.0193652\}, \\
x=100 \leftrightarrow\{0.0388411,0.387861,0.155144,0.0387859\} .
\end{gathered}
$$

The values in brackets correspond to the A, B, C, D type excitations respectively. For $x=1$ the expected rates for recoil experiments are slightly higher, but for $2<x<10$ the atomic excitations are favored.

In the case of bound electrons we should compare the results of Figs 5 and 7 . We observe that in the recoil experiments, in addition to $x$, the rates depend on the atom, as shown in the figure, so we will only present limits on the rates for a range of $x$. For $x<5$, rates $R \geq 10^{-6} \mathrm{y}^{-1}$ are not possible. For $5<x<10$ the expected rates are in the range $10^{-6}<R<10^{-5} \mathrm{y}^{-1}$. For $10<x<20,10^{-5}<R<10^{-4} \mathrm{y}^{-1}$ and for $20<x<30,10^{-4}<R<10^{-3} \mathrm{y}^{-1}$. Rates of of about $0.1 \mathrm{y}^{-1}$ do not appear before $x=100$.

The atomic excitation rates are favored for values of $2<x<100$.

For values of $x>100$ the recoil experiments are preferred, since, then, the binding electron energy becomes unimportant and all electrons can participate..

\section{DISCUSSION}

In the present paper we examined the possibility of detecting light WIMPs by exploiting their possible interactions with electrons. We calculated the event rates of various processes assuming a target with $10^{24}$ atoms

For WIMPs in the mass range of the electron mass, the energy that can be transferred to the electron is in the $\mathrm{eV}$ region. It is, therefore, very difficult for electrons to escape their binding and be ejected. Detectors utilizing Fermi-degenerate materials like superconductors [61], have recently been suggested. In this case the energy required is essentially the gap energy of about $1.5 k T_{c}$ which is in the meV region, i.e the electrons are essentially free. The WIMP density in our vicinity becomes quite high due to their small mass and the WIMP-electron cross section may be quite enhanced for scalar WIMPs. The event rates can be reasonably high for such WIMPs, but the amount of energy deposited in the detector is quite small. Detection of light WIMPs may become possible, even if the detectors operate with a non zero energy threshold. Thus, e.g., in the case of the recently proposed experiment using superconducting nanowires [62], the threshold of $0.8 \mathrm{eV}$ can be overcome for WIMPs with $x \geq 2$

Even in the case of heavier WIMPs, with masses up to 30 times the electron mass, only electrons with small binding can be ejected and, thus, the expected rate for electron recoils is quite small, $R \leq 10^{-3}$ per year, depending on the target, see table II. For still heavier WIMPs, detection rate rises quite fast and 0.1 events per year are expected for $x=100$ and keeps rising with increasing $x$.

We have also seen that it may be possible to detect light WIMPs via atomic excitations due to the well known electron spin interactions of the axial current. Thus, using a detector at low temperature in a magnetic field, a variety of transitions between the magnetic sub-states may arise, namely $\Delta m_{s}=1$ in the same shell or $\Delta m_{s}=0, \pm 1$ between the spin-orbit partners. For atoms with possible $2 \mathrm{p}$ and $3 \mathrm{~d}$ transitions, e.g., rates up to $8 \times 10^{-3}$ and $4 \times 10^{-2}$ events per year are expected, for $\mathrm{x}=2$ and $x=10$ respectively. In general the obtained events are higher than those expected in the recoil experiments for $x<100$. An additional advantage is that one can benefit from the very characteristic experimental signature of atomic excitations, namely the de-excitation signals.

\section{Acknowledgments}

J.D.V is happy to acknowledge support of the CERN theory division, during the last stages of this work as well as support by the National Experts Council of China via a "Foreign Master" grant while at Nanjing University, where this work began. Special thanks to Professor S. Cohen of te University of Ioannina for very fruitful discussions. 


\section{References}

[1] S. Hanary et al., Astrophys. J. 545, L5 (2000).

[2] J. Wu et al., Phys. Rev. Lett. 87, 251303 (2001).

[3] M. Santos et al., Phys. Rev. Lett. 88, 241302 (2002).

[4] P. D. Mauskopf et al., Astrophys. J. 536, L59 (2002).

[5] S. Mosi et al., Prog. Nuc.Part. Phys. 48, 243 (2002).

[6] N. W. Halverson et al., Astrophys. J. 568, 38 (2002).

[7] G. F. Smoot et al., Astrophys. J. 396, L1 (1992), the COBE Collaboration.

[8] D. N. Spergel et al., Astrophys. J. Suppl. 148, 175 (2003).

[9] A. H. Jaffe et al., Phys. Rev. Lett. 86, 3475 (2001).

[10] D. Spergel et al., Astrophys. J. Suppl. 170, 377 (2007), arXiv:astro-ph/0603449v2].

[11] The Planck Collaboration, A.P.R. Ade et al, Astron. Astrophys. 571 (2014), A16 arXiv:1303.5076 [astro-ph.CO].

[12] P. Ullio and M. Kamioknowski, JHEP 0103, 049 (2001).

[13] M. Battaglieri et al, US Cosmic Visions: New Ideas in Dark Matter 2017: Community Report, arXiv:1707.04591 [hep-ph].

[14] J. D. Vergados, H. Ejiri, and K. Savvidy, Nuc. Phys. B 877, 36 (2013), arXiv:1307.4713 (hep-ph).

[15] J. D. Vergados, F. T. A. III, P. Pirinen, P. C. Srivastava, M. Kortelainen, and J. Suhonen, Phys. Rev. D 92, 015015 (2015), arXiv:1504.02803 (hep-ph)

[16] J. D. Lewin and P. F. Smith, Astropart. Phys. 6, 87 (1996).

[17] M. W. Goodman and E. Witten, Phys. Rev. D 31, 3059 (1985).

[18] A. Drukier, K. Freeze, and D. Spergel, Phys. Rev. D 33, 3495 (1986).

[19] J. R. Primack, D. Seckel, and B. Sadoulet, Ann. Rev. Nucl. Part. Sci. 38, 751 (1988).

[20] A. Gabutti and K. Schmiemann, Phys. Lett. B 308, 411 (1993).

[21] R. Bernabei, Riv. Nouvo Cimento 18 (5), 1 (1995).

[22] D. Abriola et al., Astropart. Phys. 10, 133 (1999), arXiv:astro-ph/9809018.

[23] F. Hasenbalg, Astropart. Phys. 9, 339 (1998), arXiv:astro-ph/9806198.

[24] J. D. Vergados, Phys. Rev. D 67, 103003 (2003), hep-ph/0303231.

[25] A. Green, Phys. Rev. D 68, 023004 (2003), ibid: D 69 (2004) 109902; arXiv:astro-ph/0304446

[26] C. Savage, K. Freese, and P. Gondolo, Phys. Rev. D 74, 043531 (2006), arXiv:astro-ph/0607121.

[27] P. J. Fox, J. Kopp, M. Lisanti and N. Weiner, A CoGeNT Modulation Analysis, arXiv:1107.0717 (astro-ph.CO).

[28] K. Abe et al., Astropart. Phys. 31, 290 (2009), arXiv:v3 [physics.ins-det]0809.4413v3 [physics.ins-det].

[29] T. Bruch et al., Phys. Rev. Lett 102, 011301 (2009), arXiv:0802.3530.

[30] E. Armengaud et al., Phys. Lett. B 702, 329 (2011), arXiv:1103.4070v3 [astro-ph.CO].

[31] S. C. Kim et al., Phys. Rev. Lett. 108, 181301 (2012), for the KIMS collaboration.

[32] M. Felizardo et al., Phys. Rev. Lett. 108, 201302 (2012), the SIMPLE collaboration, see also Erratum: Phys. Rev. D 90, 079902 (2014), arXiv:1003.2987 [astro-phCO].

[33] S. Archambault et al., Phys. Lett. B 711, 153 (2012), the PICASSO collaboration.

[34] R. Bernabei et al., Eur. Phys. J. C 73, 2648 (2013), [DAMA/LIBRA phase 1]; arXiv:1308.5109 (astro-GA.).

[35] The CRESST Experiment: Recent Results and Prospects, P.Di Stefano, et al, arXiv:hep-ex/0011064; The CRESST Collaboration, talk presented at IBS - MultiDark Joint Focus Program, Daejeon, s. Korea, 1021 October 2014.

[36] E. Aprile et al., Phys. Rev. Lett. 119, 181301 (2017), [XENON100 Collaboration]; arXiv:1705.06655.

[37] D. Akerib et al., Phys. Rev. Lett. 112, 091303 (2014), arXiv:1310.8214.

[38] Sunghyun Kang, Stefano Scopel, Gaurav Tomar, JongHyun, Present and projected sensitivities of Dark Matter direct detection experiments to effective WIMP-nucleus couplings, arXiv:1805.06113

[39] LUX-ZEPLIN Collaboration, D. S. Akerib et al., Projected WIMP sensitivity of the LUX-ZEPLIN (LZ) dark matter experiment, arXiv:1802.06039.

[40] R. Essig, J. Mardon, and T. Volansky, Phys. Rev. D 85, 076007 (2012).

[41] R. Essig, A. Manalaysay, J. Mardon, and T. Volansky, Phys. Rev. Lett 109, 021301 (2012).

[42] R. Essig, T. Volansky, and T. T. Yu, Phys. Rev D 96, 043017 (2017), arXiv:1703.00910 [hep-ph].

[43] K. Schutz and K. M. Zurec, Phys. Rev. Lett 117, 121302 (2016), arXiv:1604.0820.

[44] C. Blanco, J.I. Collar, Y. Kahn, and B. Lillard, Dark Matter-Electron Scattering from Aromatic Organic Targets arXiv:1912.02822 [hep-ph].

[45] J. I. Collar, Phys. Rev D 98, 0230057 (2018), arXiv:1805.02646 [astro-ph.CO].

[46] Y. Hochberg, Y. Kahn, M. Lisanti, C. G. Tully, and K. M. Zurek, Phys. Lett. B 772, 239 (2017).

[47] S. Derenzo, R. Essig, A. Massari, A. Soto, and T.-T. Yu, Phys. Rev. D 96, 016026 (2017), arXiv:1703.01009 (astro-ph.CO), (hep-ex).

[48] G. Kopidakis, J.D. Vergasos et al, to be published.

[49] V. Oikonomou, J. Vergados, and C. C. Moustakidis, Nuc. Phys. B 773, 19 (2007). 
[50] C. Boehm and P. Fayet, Nucl.Phys. B 683, 29 (2004), arXiv:hep-ph/0305261.

[51] E. Ma, Phys. Rev. D 73, 077301 (2006), arXiv:hep-ph/0601225.

[52] V. Silveira and A. Zee, Phys. Lett. B 161, 136 (1985).

[53] D. Holz and A. Zee, Phys. Lett. B 517, 239 (201).

[54] M. Bento, O. Berolami, and R. Rosefeld, Phys. lett. B 518, 276 (2001).

[55] M. Bento, O. Berolami, R. Rosefeld, and L. Teodoro, Phys. Rev. D 62, 041302 (2000).

[56] Y.-K. E. Cheung and J. D. Vergados, JCAP 1502, 014 (2015), 1410.5710.

[57] C. Li, R. H. Brandenberger, and Y.-K. E. Cheung, Phys. Rev. D90, 123535 (2014), 1403.5625.

[58] Y.-K. E. Cheung, J. U. Kang, and C. Li, JCAP 1411, 001 (2014), 1408.4387.

[59] J. Vergados, C. C. Moustakidis, Y.-K. E. Cheung, H. Ejiri, Y. Kim, and J. yen Lee, AHEP 2018, 6257198 (2018).

[60] G. Angloher et al., Eur. Phys. J C 74, 12 (2014), astro-ph/1407.3146.

[61] Y. Hocberg,M. Pyle, Y. Zhao and M, Zurek, Detecting superlight Dark Matter with Fermi Degenerate Materials, arXiv:1512,04533 [hep-ph].

[62] Y. Hochberg, I. Charaev, S. W. N. V. Verma, M. Colangelo, and K. K. Berggren, Phys. Rev Lett. 123, 151802 (2019), arXiv:1903.05101 [hep-ph].

[63] N. Kurinsky, T. C. Yu, Y. Hochberg, and B. Cabrera, Phys. Rev D 99, 123005 (2019), arXiv:1901.07569 [hep-ex].

[64] F. Larkins, At. Data and Nucl. Data Tables 20, 313 (1977).

[65] K.D. Sevier, Low Energy Electron Spectrometry, Wiley- Interscience, New York (1972).

[66] P. F.T and M. Freedman, J. Phys. Chem. Ref. Data 7, 1267 (1978).

[67] P. Sikivie, Phys. Rev. Lett. 113, 201301 (2014).

[68] F.T. Avignone III, R. J. Creswick and J. D. Vergados, Axion Detection via Atomic Excitations, arXiv:1801.02072 (hep-ph). 


\section{APPENDIX}

In this section we present the angular momentum parameters needed in evaluating the atomic excitation rates.

TABLE III: the coefficients $\left(C_{j_{1}, m_{1}, j_{2}, m_{2}, \ell}\right)^{2}$ connecting via the spin operator a given initial state $|i\rangle=\left|n \ell, j_{1}, m_{1}\right\rangle$ with all possible states $|f\rangle=\left|n \ell, j_{2}, m_{2}\right\rangle$, for $\ell=0,1$. Note s-states are favored.

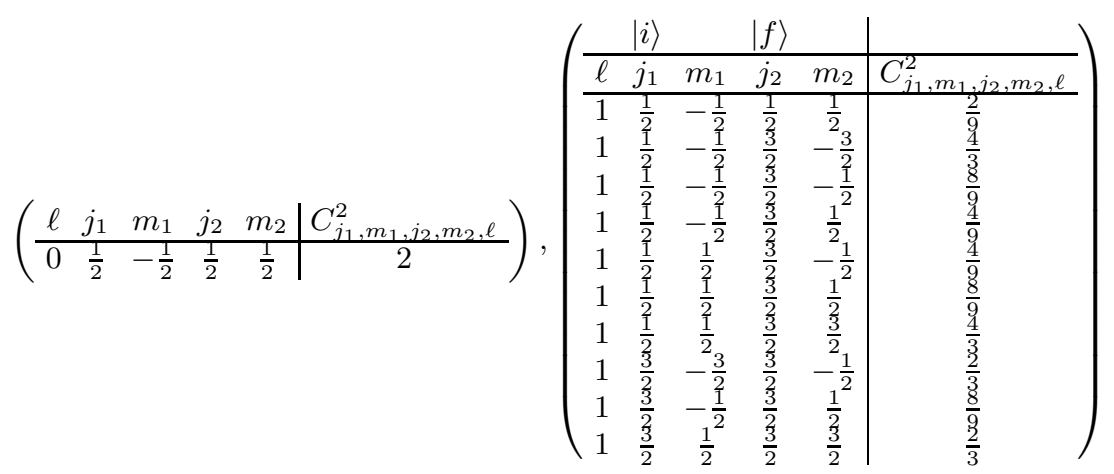

TABLE IV: The same as in table III, the coefficients $\left(C_{j_{1}, m_{1}, j_{2}, m_{2}, \ell}\right)^{2}$ for $\ell=2$

$\left(\begin{array}{ccccc|c}\multicolumn{2}{c}{|i\rangle} & \multicolumn{1}{l|}{|f\rangle} & \\ \hline \ell & j_{1} & m_{1} & j_{2} & m_{2} & C_{j_{1}, m_{1}, j_{2}, m_{2}, \ell}^{2} \\ \hline 2 & \frac{3}{2} & -\frac{3}{2} & \frac{3}{2} & -\frac{1}{2} & \frac{6}{25} \\ 2 & \frac{3}{2} & -\frac{3}{2} & \frac{5}{2} & -\frac{5}{2} & \frac{8}{5} \\ 2 & \frac{3}{2} & -\frac{3}{2} & \frac{5}{2} & -\frac{3}{2} & \frac{16}{25} \\ 2 & \frac{3}{2} & -\frac{3}{2} & \frac{5}{2} & -\frac{1}{2} & \frac{4}{25} \\ 2 & \frac{3}{2} & -\frac{1}{2} & \frac{3}{2} & \frac{1}{2} & \frac{8}{25} \\ 2 & \frac{3}{2} & -\frac{1}{2} & \frac{5}{2} & -\frac{3}{2} & \frac{24}{25} \\ 2 & \frac{3}{2} & -\frac{1}{2} & \frac{5}{2} & -\frac{1}{2} & \frac{24}{25} \\ 2 & \frac{3}{2} & -\frac{1}{2} & \frac{5}{2} & \frac{1}{2} & \frac{12}{25} \\ 2 & \frac{3}{2} & \frac{1}{2} & \frac{3}{2} & \frac{3}{2} & \frac{6}{25} \\ 2 & \frac{3}{2} & \frac{1}{2} & \frac{5}{2} & -\frac{1}{2} & \frac{12}{25} \\ 2 & \frac{3}{2} & \frac{1}{2} & \frac{5}{2} & \frac{1}{2} & \frac{24}{25} \\ 2 & \frac{3}{2} & \frac{1}{2} & \frac{5}{2} & \frac{3}{2} & \frac{24}{25} \\ 2 & \frac{3}{2} & \frac{3}{2} & \frac{5}{2} & \frac{1}{2} & \frac{4}{25} \\ 2 & \frac{3}{2} & \frac{3}{2} & \frac{5}{2} & \frac{3}{2} & \frac{16}{25} \\ 2 & \frac{3}{2} & \frac{3}{2} & \frac{5}{2} & \frac{5}{2} & \frac{8}{5} \\ 2 & \frac{5}{2} & -\frac{5}{2} & \frac{5}{2} & -\frac{3}{2} & \frac{2}{5} \\ 2 & \frac{5}{2} & -\frac{3}{2} & \frac{5}{2} & -\frac{1}{2} & \frac{16}{25} \\ 2 & \frac{5}{2} & -\frac{1}{2} & \frac{5}{2} & \frac{1}{2} & \frac{18}{25} \\ 2 & \frac{5}{2} & \frac{1}{2} & \frac{5}{2} & \frac{3}{2} & \frac{16}{25} \\ 2 & \frac{5}{2} & \frac{3}{2} & \frac{5}{2} & \frac{5}{2} & \frac{5}{5}\end{array}\right)$

